HD-THEP-93-41

Sw 9408

\title{
EXACT EVOLUTION EQUATION FOR SCALAR ELECTRODYNAMICS
}

\author{
M. Reuter \\ DESY, Notkestraße 85, D-22603 Hamburg \\ C. Wetterich \\ Institut für Theoretische Physik \\ Universität Heidelberg \\ Philosophenweg 16, D-69120 Heidelberg
}

\begin{abstract}
:
We describe scalar quantum electrodynamics by a gauge-invariant effective average action $\Gamma_{k}$. This obtains by integrating out the quantum fluctuations with "momenta" larger than $k$ in a gauge-invariant way. Our method can deal successfully with the infrared problems of perturbation theory in less than four dimensions or at high temperature. The dependence of $\Gamma_{k}$ on the infrared cutoff $k$ is given by an exact evolution equation.
\end{abstract}





\section{Introduction}

Quantum electrodynamics has been the prototype for the perturbative loop expansion in quantum field theory. Theoretical predictions based on perturbation theory have been tested with an impressive accuracy. It is widely believed that theoretical predictions hold with a similar accuracy if the electron is replaced by a massive charged scalar field. There remain, however, some questions on the validity of the perturbative picture of the transition to the phase with spontaneous symmetry breaking [1] - strictly speaking the perturbative series cannot converge for the computation of the effective scalar potential in the region where its perturbative approximation is not convex. Compared to the four-dimensional theory at zero temperature the status of perturbation theory becomes dramatically more problematic in the three-dimensional theory which should describe the phase transition of superconductors or in the four-dimensional theory at nonvanishing temperature which is often considered as a prototype for the description of the electroweak phase transition in the early universe.

In (scalar) quantum electrodynamics (QED) at nonvanishing temperature the perturbative expansion is plagued by severe infrared problems [2]. For Green functions with momenta much smaller than the temperature, the structure of the theory is essentially determined by three-dimensional QED. The infrared behaviour for vanishing particle masses reflects then the corresponding strong infrared divergences in three-dimensional perturbation theory. For the three-dimensional theory in the symmetric phase the one-loop correction to the quartic scalar coupling diverges $\sim k^{-1}$, with $k$ an appropriate infrared cutoff. Usually, one tries to remedy this situation by a "resummation of diagrams". This induces a "plasma mass" $M_{p l}$ for the photon which acts as an effective infrared cutoff. For sufficiently large plasma mass the corrections $\sim M_{p l}^{-1}$ remain small. Conditions for the reliability of resummed perturbation theory have been discussed extensively in ref. [3]. Another infrared divergence $\sim k^{-1}$ arises for the one-loop correction to the gauge coupling if the scalar mass $m$ vanishes. Near the phase transition $m$ may be very small and corrections proportional $\mathrm{m}^{-1}$ can be substantial. This effect is commonly not yet included in high temperature resummed perturbation theory.

A new method for dealing nonperturbatively with these infrared problems is based on the concept of an average action $\Gamma_{k}$ [4]. For a computation of $\Gamma_{k}$ only quantum fluctuations with momenta $q^{2}>k^{2}$ are included such that no infrared divergences can appear for $k>0$. By following the $k$-dependence of $\Gamma_{k}$ with the help of an evolution equation one may then extrapolate to $k \rightarrow 0$. In the limit $k \rightarrow 0$ the effective average action $\Gamma_{k}$ becomes the generating functional for the 1PI Green functions, i.e. the effective action as defined usually by a Legendre transform. The vacuum properties can then be read off from $\Gamma_{k \rightarrow 0}$. It has been demonstrated in a $\varphi^{4}$-theory at high temperature that this method can indeed give an accurate description of the infrared properties of the model [5]. Even at the critical temperature for the second order phase transition of this theory, the one-loop infrared divergences $\sim k^{-1}$ appear to be artefacts of perturbation theory. The true nonanalytical aspects of the critical theory are all encoded in the critical exponents 
of the three-dimensional $\varphi^{4}$-theory. For $k>0$ the average action plays the role of a "coarse-grained action" as needed in particular for the treatment of first-order phase transitions [6]. The concept of the average action has been generalized to gauge theories [7], [8]. In particular, the running of the gauge coupling in the effective three-dimensional theory has been computed [8]. This deals successfully with the apparently diverging one-loop correction $\sim k^{-1}$ in massless gauge theories at high temperature. A bound on the reliability of high temperature perturbation theory has also been derived from these considerations [8].

It has been shown recently [9] that the $k$-dependence of the effective average action for a scalar theory can be described by an exact evolution equation. This equation has the simple form of a one-loop equation with an additional infrared cutoff term $R_{k}$ in the propagator. In contrast to the one-loop approximation all propagators and vertices are now given by appropriate functional derivatives of $\Gamma_{k}$ instead of the classical propagator $G$ and classical vertices. In analogy to the $k$ derivative of the one-loop expression $-\frac{1}{2} \operatorname{Tr} \ln G$ the exact equation reads

$$
\frac{\partial}{\partial t} \Gamma_{k}=\frac{1}{2} \operatorname{Tr}\left\{\left(\Gamma_{k}^{(2)}+R_{k}\right)^{-1} \frac{\partial}{\partial t} R_{k}\right\}
$$

Here the trace amounts to a sum over momenta and internal indices, $\Gamma_{k}^{(2)}$ is the second functional derivative of $\Gamma_{k}$ with respect to the fields (the exact inverse propagator) and $t=\ln k$. The simple form of (1.1) allows for useful perturbative truncations for its solutions. This seems to be a certain advantage as compared to earlier versions of exact renormalization group equations [10] which were usually solved approximately only by reconstructing the perturbative loop expansion. The formal relation between eq. (1.1) and the exact renormalization group equations [10] has by now been established $[11]^{1}$.

In this paper we deal with the generalization of the exact evolution equation (1.1) for the abelian Higgs model. The generalization is straightforward in a gaugefixed version where the formalism of ref. [9] can be simply applied. The price for abandoning the gauge invariance of $\Gamma_{k}$ is, however, rather high: Without gauge symmetry the number of invariants in $\Gamma_{k}$ with a given dimension increases dramatically. This makes it much more difficult to find useful truncations beyond perturbation theory. In addition, there is no immediate control of physical gauge invariance for any nonzero value of $k$. Only for $k=0$ the Ward identities encoding the symmetry properties of a gauge-invariant theory have to obeyed. To avoid these difficulties (at least partially) we employ a formulation closely related to the background field formalism [12]. This enables us to construct a gauge-invariant functional $\Gamma_{k}$. The main modification of (1.1) in this formulation is the appearance of a term reflecting a generalized $k$-dependent gauge fixing in addition to $\Gamma_{k}^{(2)}$. This ensures that propagators are well-behaved for a gauge-invariant $\Gamma_{k}$.

The effective average action for a scalar field can be represented by a functional integral where the classical action $S$ is expanded around some background field $\varphi$

\footnotetext{
${ }^{1}$ There should also exist a formal relation to the exact Schwinger-Dyson equations. This remains to be established.
} 
[13]. It reads in momentum space

$$
\exp -\Gamma_{k}[\varphi]=\int D \chi \exp -\left\{S[\varphi+\chi]-\sum_{q} \frac{\partial \Gamma_{k}}{\partial \varphi(q)} \chi(q)+\frac{1}{2} \Omega \sum_{q} R_{k}(q) \chi^{*}(q) \chi(q)\right\}
$$

Here the infrared cutoff function $R_{k}(q)$ should be $\sim k^{2}$ for $q^{2} \ll k^{2}$ and decay exponentially for $q^{2} \gg k^{2}$. ${ }^{2}$ One obviously recovers the standard functional integral representation of the effective action for $k \rightarrow 0$ where $R_{k}$ vanishes identically. This formulation can easily be generalized to the abelian Higgs model with a complex scalar field and an abelian gauge field which we split into a background field $A_{\mu}$ and a fluctuation $a_{\mu}$ :

$$
\begin{aligned}
& \exp -\Gamma_{k}[\varphi, A]=\exp -C_{k}[A] \int \mathcal{D} \chi \mathcal{D} a \exp -\{S[\varphi+\chi, A+a] \\
& \left.+\frac{1}{2 \alpha} \int d^{d} x\left(\partial^{\mu} a_{\mu}\right)^{2}-\int d^{d} x\left(\left(\frac{\delta \Gamma_{k}}{\delta \varphi}\right) \chi+\left(\frac{\delta \Gamma_{k}}{\delta A_{\mu}}-\tilde{\kappa}^{\mu}\right) a_{\mu}\right)+\Delta_{k} S\right\}
\end{aligned}
$$

The infrared cutoff $\Delta_{k} S$ can be constructed in a gauge invariant way using covariant derivatives for the scalar field and observing that the fluctuation field $a_{\mu}$ is invariant in a formulation where the background field $A_{\mu}$ transforms as a usual gauge field. (For details of $\Delta_{k} S$ see later.) The functional $\Gamma_{k}[\varphi, A]$ is then manifestly invariant under simultaneous gauge transformations of $\varphi$ and $A$. The expression (1.3) is closely related to the formulation of ref. [7], [8], where the role of the gauge-invariant normalization derminant $\exp -C_{k}[A]$ has been discussed extensively. ${ }^{3}$ In complete analogy to (1.1) we will show that the $k$-dependence of the gauge-invariant effective average action is described by an exact evolution equation

$$
\begin{aligned}
& \frac{\partial}{\partial t} \Gamma_{k}[\varphi, A]=\frac{\partial}{\partial t} C_{k}[A] \\
& +\frac{1}{2} \operatorname{Tr}\left\{\frac{\partial}{\partial t} R_{k}[A]\left(\Gamma_{k}^{(2)}[\varphi, A]+\Gamma_{\mathrm{gf}}^{(2)}[\varphi, A]+R_{k}[A]\right)^{-1}\right\}
\end{aligned}
$$

The new piece $\Gamma_{\mathrm{gf}}^{(2)}$ corresponds to the second functional derivative of a generalized $k$ dependent gauge-fixing term. It will be discussed in detail in the following sections, where we will also give an explicit definition of $C_{k}[A]$.

The evolution equation (1.4) is the starting point for a nonperturbative treatment of the abelian Higgs model. This equation will be derived in sects. 2-4. Particular emphasis is placed on the role of the background field and its relation to gauge invariance. The formal development of these sections can easily be extended to scalars and fermions with arbitrary charges provided the fermion representation is free of anomalies. In sect. 5 we specialize to a discussion of the abelian Higgs model in arbitrary dimension. We concentrate mainly on nonvanishing expectation values of the scalar fields, but the simpler case of the symmetric phase is also covered. We

\footnotetext{
${ }^{2}$ We work here on a torus with volume $\Omega$ and take $\Omega-\infty$ for the final results.

3Our formulation actually corresponds to the "improved average action" [13]. The correction $\tilde{\kappa}^{\mu}$ in the "source term" is discussed later.
} 
make a simple truncation of $\Gamma_{k}[\varphi, A]$ and derive approximative, albeit nonperturbative equations for the running of the gauge coupling, the quartic scalar coupling, and the scalar mass term (or equivalently the location of the potential minimum). These equations, which can be generalized to nonvanishing temperature similar to ref. [5], constitute the basis for a nonperturbative treatment of three-dimensional QED and four-dimensional QED at high temperature. The numerical or analytical solution of these equations is left for future work, being outside the more formal scope of the present paper. The explicit form of the evolution equations demonstrates clearly that our nonperturbative method can indeed handle the perturbative infrared problems.

\section{Evolution equation with background field}

The exact evolution equation for an abelian gauge theory with a charged scalar field can be derived in complete analogy to ref. [9], using the background field formalism. Since it can also be obtained as a special case of the more general equation for arbitrary gauge theories [14] we give here only a brief sketch of its derivation. Our starting point is the scale-dependent generating functional for the connected Green functions for a complex scalar field $\chi$ and fluctuations of the gauge field $\mathcal{A}_{\mu}$ around some arbitrarily chosen background field $\bar{A}_{\mu}, \mathcal{A}_{\mu}=\bar{A}_{\mu}+a_{\mu}$

$$
\begin{aligned}
& \exp W_{k}[J, K ; \bar{A}]=\int D \chi D a \exp -\{S[\chi, \bar{A}+a] \\
& +S_{\mathrm{gf}}[a]+\Delta_{k} S_{S}[\chi ; \bar{A}]+\Delta_{k} S_{G}[a] \\
& \left.-\int d^{d} x\left(J^{*} \chi+\chi^{*} J+K^{\mu} a_{\mu}\right)\right\}
\end{aligned}
$$

Here $S[\chi, A]$ is the gauge-invariant action for scalar and gauge fields, and $S_{\mathrm{gf}}$ plays the role of a gauge-fixing term

$$
S_{\mathrm{gf}}=\frac{1}{2 \alpha} \int d^{d} x\left(\partial^{\mu} a_{\mu}\right)^{2}
$$

The terms $\Delta_{k} S_{S}$ and $\Delta_{k} S_{G}$ introduce effective infrared cutoffs for the scalars and gauge fields, respectively. They suppress the contributions of quantum fluctuations with $q^{2}<k^{2}$ to the functional integral (2.1). We choose [7],[8],[14]

$$
\begin{aligned}
\Delta_{k} S_{S}= & \int d^{d} x \chi^{*} R_{k}^{(\mathcal{S})}\left(\mathcal{D}_{S}[\bar{A}]\right) \chi \\
\Delta_{k} S_{G}= & \frac{1}{2} \int d^{d} x a^{\mu} R_{k}^{(G)}\left(-\partial^{2}\right) a_{\mu} \\
& +\frac{1}{2} \int d^{d} x\left(\partial^{\nu} a_{\nu}\right) \frac{\alpha^{-1} \tilde{R}_{k}^{(G)}\left(-\partial^{2}\right)-R_{k}^{(G)}\left(-\partial^{2}\right)}{\left(-\partial^{2}\right)}\left(\partial^{\mu} a_{\mu}\right) \\
= & \frac{1}{4} \int d^{d} x f^{\mu \nu} \frac{R_{k}^{(G)}\left(-\partial^{2}\right)}{\left(-\partial^{2}\right)} f_{\mu \nu} \\
& +\frac{1}{2 \alpha} \int d^{d} x\left(\partial^{\nu} a_{\nu}\right) \frac{\tilde{R}_{k}^{(G)}\left(-\partial^{2}\right)}{\left(-\partial^{2}\right)}\left(\partial^{\mu} a_{\mu}\right)
\end{aligned}
$$


with $f_{\mu \nu}=\partial_{\mu} a_{\nu}-\partial_{\nu} a_{\mu}, \mathcal{D}_{S}[\bar{A}]=-\left(\partial^{\mu}+i \bar{e} \bar{A}^{\mu}\right)\left(\partial_{\mu}+i \bar{e} \bar{A}_{\mu}\right)$ and

$$
\begin{aligned}
& R_{k}^{(S)}(x)=\frac{Z_{\varphi, k}\left(x+m_{k}^{2}\right) f_{k}^{2}\left(x+m_{k}^{2}\right)}{1-f_{k}^{2}\left(x+m_{k}^{2}\right)} \\
& R_{k}^{(G)}(x)=\frac{Z_{F, k}\left(x+M_{k}^{2}\right) f_{k}^{2}\left(x+M_{k}^{2}\right)}{1-f_{k}^{2}\left(x+M_{k}^{2}\right)} \\
& \tilde{R}_{k}^{(G)}(x)=\frac{Z_{G, k} x f_{k}^{2}(x)}{1-f_{k}^{2}(x)}
\end{aligned}
$$

Here $Z_{\varphi, k} Z_{F, k}$ and $Z_{G, k}$ are appropriate wave function renormalizations and $m_{k}^{2} \geq$ $0, M_{k}^{2} \geq 0$ are mass terms which may be used to optimize the infrared cutoff. (For the symmetric phase we take $M_{k}^{2}=0$, whereas for the phase with spontaneous symmetry breaking $m_{k}^{2}=0$.) The function

$$
f_{k}(x)=\exp \left\{-a\left(\frac{x}{k^{2}}\right)^{b}\right\}
$$

specifies the precise form of the infrared cutoff, and we will concentrate in the present paper on $a=\frac{1}{2}, b=1$. In the limit $k \rightarrow 0$ the additional terms $\Delta_{k} S_{S}+\Delta_{k} S_{G}$ vanish and $W_{0}[J, K ; A]$ is the usual generating functional for the connected Green functions in the background field formalism.

The effective average action $\Gamma_{k}[\varphi, \bar{a} ; \bar{A}]$ is defined by a Legendre transform

$$
\begin{gathered}
\tilde{\Gamma}_{k}[\varphi, \bar{a} ; \bar{A}]=-W_{k}[J, K ; \bar{A}]+\int d^{d} x\left(J^{*} \varphi+\varphi^{*} J+K^{\mu} \bar{a}_{\mu}\right) \\
\Gamma_{k}[\varphi, \bar{a} ; \bar{A}]=\tilde{\Gamma}_{k}[\varphi, \bar{a} ; \bar{A}]-\Delta_{k} S_{S}[\varphi ; \bar{A}]-\Delta_{k} S_{G}[\bar{a}]
\end{gathered}
$$

with

$$
\begin{array}{ll}
\varphi=\frac{\delta W_{k}}{\delta J^{*}}, & \bar{a}_{\mu}=\frac{\delta W_{k}}{\delta K^{\mu}} \\
J^{*}=\frac{\delta \tilde{\Gamma}_{k}}{\delta \varphi}, & K^{\mu}=\frac{\delta \tilde{\Gamma}_{k}}{\delta \bar{a}_{\mu}}
\end{array}
$$

In the limit $k \rightarrow 0$ the effective average action becomes the generating functional for the 1PI Green functions for the scalars $\chi$ and photons $a_{\mu}$. We combine

$$
A_{\mu}=\bar{A}_{\mu}+\bar{a}_{\mu}
$$

and define a functional depending on two gauge fields $A$ and $\bar{A}$

$$
\Gamma_{k}[\varphi, A, \bar{A}]=\Gamma_{k}[\varphi, \bar{a} ; \bar{A}]
$$

Using a version of gauge invariance where $\bar{A}_{\mu}$ transforms inhomogeneously whereas the fluctuation $a_{\mu}$ remains invariant, it is easy to show that $\Gamma_{k}[\varphi, A, \bar{A}]$ is gaugeinvariant under simultaneous gauge transformations of $p$ and both gauge fields $A$ and $\bar{A}$. For the derivation of the exact evolution equation it is convenient to use

$$
\psi=\left(\varphi, \varphi^{*}, \bar{a}_{\mu}\right)
$$


such that

$$
\Delta_{k} S_{S}+\Delta_{k} S_{G}=\frac{1}{2} \int d^{d} x \psi_{m}\left(R_{k}\right)_{n}^{m} \psi^{n}=\frac{1}{2} \operatorname{Tr}\left\{R_{k}(\psi \otimes \psi)\right\}
$$

(Instead of $\varphi$ and $\varphi^{*}$ we may also use two real scalar fields.) Since in (2.1) the only dependence on the scale $k$ arises from a simple term quadratic in the fields, it is straightforward to express the scale dependence of $\Gamma_{k}$ in terms of the twopoint function $G_{k}$ (the second functional derivative of $W_{k}$ ). One finds [14] the exact evolution equation $(t=\ln k)$

$$
\begin{aligned}
& \frac{\partial}{\partial t} \Gamma_{k}[\varphi, A, \bar{A}]=\frac{1}{2} \operatorname{Tr}\left\{G_{k} \frac{\partial}{\partial t} R_{k}\right\} \\
& =\frac{1}{2} \operatorname{Tr}\left\{\left(\frac{\partial}{\partial t} R_{k}[\bar{A}]\right)\left(\Gamma_{k}^{(2)}[\varphi, A, \bar{A}]+R_{k}[\bar{A}]\right)^{-1}\right\}
\end{aligned}
$$

Here $\Gamma_{k}^{(2)}[\varphi, A, \bar{A}]$ is the matrix of second functional derivatives of $\Gamma_{k}$ with respect to $\varphi$ and $A$ for fixed $\bar{A}$. Our equation expresses the scale dependence of $\Gamma_{k}$ in terms of the exact inverse propagator $\Gamma_{k}^{(2)}$ in presence of arbitrary fields $\varphi$ and $A$, whereby an infrared cutoff $R_{k}$ is added. The "initial value" for the solution of the evolution equation can be determined for $k \rightarrow \infty$. In this limit all eigenvalues of $R_{k}$ diverge and the classical approximation to $W_{k}(2.1)$ becomes exact. One finds

$$
\lim _{k \rightarrow \infty} \Gamma_{k}[\varphi, A, \bar{A}]=S[\varphi, A]+S_{\mathrm{gf}}[A-\bar{A}]
$$

Solving the exact evolution equation (2.15) with initiai condition (2.16) for $k \rightarrow 0$ amounts to a complete solution of the theory. All Green functions can be obtained from functional derivatives of $\Gamma_{0}[\varphi, A, \bar{A}]$ with respect to $\varphi$ and $A$ at fixed $\bar{A}$.

A somewhat disturbing feature in this formulation is the appearance of the background field $\bar{A}$ in addition to the photon field $A$. In presence of two gauge fields, gauge invariance looses much of its restrictive character since $A_{\mu}-\bar{A}_{\mu}$ is gaugeinvariant.

One is therefore tempted to identify $\bar{A}$ and $A$ in order to obtain a gauge-invariant functional depending only on the photon field $A$

$$
\begin{gathered}
\bar{\Gamma}_{k}[\varphi, A]=\Gamma_{k}[\varphi, A, A] \\
\Gamma_{k}[\varphi, A, \bar{A}]=\bar{\Gamma}_{k}[\varphi, A]+\Gamma_{k}^{\text {gauge }}[\varphi, A, \bar{A}]
\end{gathered}
$$

Here $\Gamma_{k}^{\text {gauge }}$ vanishes for $A=\bar{A}$, and comparison with (2.16) suggests the interpretation of $\Gamma_{k}^{\text {gauge }}$ as a generalized scale-dependent gauge-fixing term. This interpretation can be substantiated by decomposing the fields $A, \bar{A}, a, \bar{a}$ into transversal and longitudinal components:

$$
\begin{aligned}
& A_{L_{\mu}}=\frac{\partial_{\mu} \partial^{\nu}}{\partial^{2}} A_{\nu} \\
& A_{T_{\mu}}=A_{\mu}-A_{L_{\mu}}
\end{aligned}
$$


We observe that the gauge transformation acts only on the longitudinal components $A_{L_{\mu}}, \bar{A}_{L \mu}$ whereas the transversal components are invariant. Also the gauge-fixing term (2.2) only involves the longitudinal component $a_{L_{\mu}}$. For $k=0$ the functional $W_{0}+\int d^{d} x K^{\mu} \bar{A}_{\mu}$ is invariant under the shift

$$
\bar{A}_{T \mu} \rightarrow \bar{A}_{T \mu}+v_{T \mu}
$$

with an arbitrary transversal vector field $v_{T \mu}$. This follows immediately from the invariance of the functional integral (2.1) with respect to a shift in the splitting between $\bar{A}_{T_{\mu}}$ and $a_{T \mu}$ :

$$
\begin{aligned}
\bar{A}_{T \mu} & \rightarrow \bar{A}_{T \mu}+v_{T \mu} \\
a_{T \mu} & \rightarrow a_{T \mu}-v_{T \mu}
\end{aligned}
$$

(This holds except for the term $\sim K$ which we have accounted for by an additional term). As a consequence of the shift invariance (2.20), $W_{0}+\int d^{d} x K^{\mu} \bar{A}_{\mu}$ only depends on the longitudinal component $\bar{A}_{L \mu}$ and $\Gamma_{0}$ is invariant under the shift

$$
\begin{aligned}
\bar{A}_{T \mu} & \rightarrow \bar{A}_{T \mu}+v_{T \mu} \\
\bar{a}_{T \mu} & \rightarrow \bar{a}_{T \mu}-v_{T \mu}
\end{aligned}
$$

We conclude that $\Gamma_{0}$ only depends on $A_{T_{\mu}}, \bar{a}_{L \mu}$ and $\bar{A}_{L \mu}$, but not separately on $\bar{a}_{T \mu}$ and $\bar{A}_{T \mu}$

$$
\begin{aligned}
& \Gamma_{0}\left[\varphi, \bar{a}_{T \mu}, \bar{a}_{L_{\mu}} ; \bar{A}_{T_{\mu}}, \bar{A}_{L \mu}\right] \\
= & \Gamma_{0}\left[\varphi, 0, \bar{a}_{L \mu} ; A_{T_{\mu}}, \bar{A}_{L \mu}\right] \\
= & \bar{\Gamma}_{0}[\varphi, A]+\Gamma_{0}^{\text {gauge }}\left[\varphi, A, A-\bar{a}_{L}\right] \\
= & \bar{\Gamma}_{0}[\varphi, A]+\Gamma_{0}^{\text {gauge }}\left[\varphi, \bar{a}_{L} ; \bar{A}_{T}+\bar{a}_{T}, \bar{A}_{L}\right]
\end{aligned}
$$

Therefore $\Gamma_{0}^{\text {gauge }}$ vanishes for $\bar{a}_{L}=0$ and plays indeed the role of a gauge-fixing term.

We may expand

$$
\Gamma_{k}^{\text {gauge }}=\int d^{d} x\left\{-\kappa^{\mu}[\varphi, \bar{A}] \bar{a}_{\mu}+\frac{1}{2} \bar{a}^{\nu} \bar{Q}_{\nu}^{\mu}[\varphi, \bar{A}] \bar{a}_{\mu}+\ldots\right\}
$$

with $\kappa^{\mu}$ and $\bar{Q}_{\nu}{ }^{\mu}$ gauge-invariant operators depending on $\varphi, \bar{A}$ and $k$, e.g.

$$
\kappa^{\mu}[\varphi, \bar{A}]=-{\frac{\delta \Gamma_{k}^{\text {gauge }}[\varphi, A, \bar{A}]}{\delta A_{\mu}}}_{\mid A=\bar{A}}=\left.\frac{\delta \Gamma_{k}[\varphi, A, \bar{A}]}{\delta \bar{A}_{\mu}}\right|_{\mid A=\bar{A}}
$$

and observe that all terms in (2.24) must involve at least one power of the longitudinal component $\bar{a}_{L_{\mu}}$ for $k \rightarrow 0$. In principle $\Gamma_{k}^{\text {gauge }}$ gives a contribution to the Green functions at $k=0$. It also plays an important role in the exact evolution equation. With

$$
\Gamma_{k}^{(2)}[\varphi, A, \bar{A}]=\bar{\Gamma}_{k}^{(2)}[\varphi, A]+\Gamma_{k}^{\text {gauge(2) }}[\varphi, A, \bar{A}]
$$

(where the second functional derivative $\Gamma_{k}^{\text {gauge(2) }}$ is evaluated at fixed background field $\bar{A}$ ) we observe that $\bar{\Gamma}_{k}^{(2)}$ alone would not provide a well behaved propagator for the longitudinal gauge boson. This only arises through the gauge-fixing term $\Gamma_{k}^{g a u g e(2)}$. 


\section{Background field dependence}

The generalized gauge-fixing term $\Gamma_{k}^{\text {gauge }}(2.18)$ is closely related to the dependence of $\Gamma_{k}[\varphi, A, \bar{A}]$ on the background field $\bar{A}$ (cf. (2.25). (If $\Gamma_{k}[\varphi, A, \bar{A}]$ were independent of $\bar{A}$ the contribution $\Gamma_{k}^{\text {gauge }}$ would vanish.) In this section we study in more quantitative detail the dependence of $\Gamma_{k}[\varphi, A, \bar{A}]$ on the background field $\bar{A}$. We are interested in the quantity $\kappa[\varphi, A, \bar{A}]$ which generalizes (2.25)

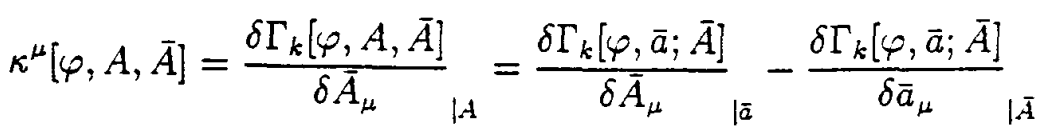

Observing

we obtain

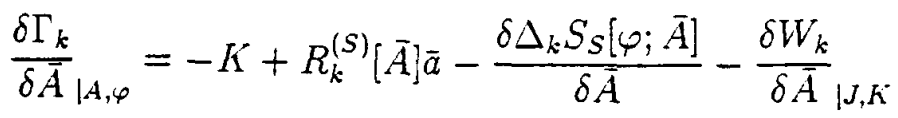

$$
\frac{\delta \Gamma_{k}}{\delta \bar{A}_{\mu \mid A, \varphi}}=\frac{1}{2} \operatorname{Tr}\left\{\left(\frac{\delta R_{k}}{\delta \bar{A}_{\mu}}\right)\left(\Gamma_{k}^{(2)}+R_{k}\right)^{-1}\right\}+\frac{1}{\alpha} \partial^{\mu} \partial_{\nu} \bar{a}^{\nu}
$$

The derivation of eq. (3.2) is analogous to the derivation of the exact evolution equation (2.15), and we note the close resemblance of the structure of both equations. The last part in eq. (3.2) can be derived from a gauge-fixing part in $\Gamma_{k}[\varphi, A, \bar{A}]$ :

$$
\begin{aligned}
\Gamma_{\mathrm{gff}} & =\frac{1}{2 \alpha} \int d^{d} x\left(\partial_{\mu} \bar{a}^{\mu}\right)^{2} \\
& =-\frac{1}{2 \alpha} \int d^{d} x\left(A_{\mu}-\bar{A}_{\mu}\right) \partial^{\mu} \partial_{\nu}\left(A^{\nu}-\bar{A}^{\nu}\right) \\
\frac{\delta \Gamma_{\mathrm{gf}}}{\delta \bar{A}_{\mu \mid A, \varphi}} & =\frac{1}{\alpha} \partial^{\mu} \partial_{\nu}\left(A^{\nu}-\bar{A}^{\nu}\right)
\end{aligned}
$$

Note that $\Gamma_{\mathrm{gf}}$ is independent of $k$. At this point there is no $k$-dependence of the gauge-fixing parameter $\alpha$. We observe that $\Gamma_{\mathrm{gf}}$ only contributes to $\Gamma_{k}^{\text {gauge }}$ in (2.18) and leaves $\bar{\Gamma}_{k}$ unaffected. It gives no contribution to $\kappa^{\mu}$, but it contributes to $\bar{Q}$ (2.24). In consequence, it only adds the usual ( $k$-independent) gauge fixing piece for the $(A, A)$-component of $\Gamma_{k}^{(2)}$, guaranteeing that the momentum integral in (2.15) is well defined. The $\bar{A}$ dependence of the remaining piece

$$
\begin{aligned}
\hat{\Gamma}_{k}^{\text {gauge }}[\varphi, A, \bar{A}] & \equiv \Gamma_{k}[\varphi, A, \bar{A}]-\bar{\Gamma}_{k}[\varphi, A]-\Gamma_{\mathrm{gf}}[A, \bar{A}] \\
& =\Gamma_{k}^{\text {gauge }}[\varphi, A, \bar{A}]-\Gamma_{\mathrm{gf}}[A, \bar{A}]
\end{aligned}
$$

arises only through the $\bar{A}$-dependent infrared cutoff for the scalar field $\Delta_{k} S_{\mathrm{S}}(2.3)$. This piece vanishes for $k \rightarrow 0$ and we conclude

$$
\begin{aligned}
\lim _{k \rightarrow 0} \Gamma_{k}[\varphi, A, \bar{A}] & =\Gamma[\varphi, A]+\Gamma_{\mathrm{gf}}[A, \bar{A}] \\
\Gamma[\varphi, A] & =\lim _{k \rightarrow 0} \bar{\Gamma}_{k}[\varphi, A]
\end{aligned}
$$

Due to the particularly simple form of $\Gamma_{\mathrm{gf}}$, which only contributes to the two-point function of the longitudinal gauge boson, all Green functions can be easily obtained 
by functionally differentiating $\Gamma[\varphi, A]$. For the abelian theory $\Gamma[\varphi, A]$ is the generating functional for the gauge-invariant 1 PI Green functions.

For nonvanishing $k$ the piece $\hat{\Gamma}_{k}^{\text {gauge }}$ is still present. It is not needed to make propagators well behaved, but it arises as a necessary consequence of gauge invariance of the infrared cutoff for the scalar fields. Several comments are in order here:

i) We can write

$$
\begin{aligned}
{\frac{\delta \hat{\Gamma}_{k}^{\text {gauge }}}{\delta \bar{A}}=}_{l \varphi, A} & \frac{1}{2} \operatorname{Tr}\left\{\frac{\delta R_{k}}{\delta \bar{A}}\left(\Gamma_{k}^{(2)}+R_{k}\right)^{-1}\right\} \\
= & \frac{1}{2} \frac{\delta}{\delta \bar{A}} \operatorname{Tr} \ln \left(\Gamma_{k}^{(2)}+R_{k}\right) \\
& -\frac{1}{2} \operatorname{Tr}\left\{\left(\frac{\delta}{\delta \bar{A}} \Gamma_{k}^{(2)}[\varphi, A, \bar{A}]\right)\left(\Gamma_{k}^{(2)}+R_{k}\right)^{-1}\right\}
\end{aligned}
$$

so that this expression simplifies considerably in the limit where $\Gamma_{k}^{(2)}[\varphi, A, \vec{A}]$ becomes independent of $\bar{A}$.

ii) We note that $R_{k}$ is a function of $\mathcal{D}_{\mathrm{S}}[\bar{A}]=-D^{\mu}[\bar{A}] D_{\mu}[\bar{A}]$ which vanishes exponentially for eigenvalues of $\mathcal{D}_{\mathrm{S}}[\bar{A}]$ much bigger than $k^{2}$. For large eigenvalues of $\mathcal{D}_{\mathrm{S}}$ also $\delta R_{k} / \delta \bar{A}$ decreases exponentially and the integral (3.6) is well defined in the ultraviolet. Its infrared finiteness follows from $\lim _{q^{2} \rightarrow 0} R_{k}=$ const.

iii) The transformation properties of $\Gamma_{k}[\varphi, A, \bar{A}]$ under gauge transformations acting only on $\varphi$ and $A$ with fixed background field $\bar{A}$ follow directly from eq. (3.2). Denoting an infinitesimal gauge transformation by $\delta_{\theta}$, one uses

$$
\delta_{\theta} \Gamma_{k}[\varphi, A, \bar{A}]=\frac{\delta \Gamma_{k}}{\delta \varphi} \delta_{\theta} \varphi+\frac{\delta \Gamma_{k}}{\delta A} \delta_{\theta} A+\frac{\delta \Gamma_{k}}{\delta \bar{A}} \delta_{\theta} \bar{A}=0
$$

and obtains for the gauge dependence of $\Gamma_{k}$ at fixed $\bar{A}$

$$
\begin{aligned}
\delta_{\theta}^{\prime} \Gamma_{k}[\varphi, A, \bar{A}] \equiv & \frac{\delta \Gamma_{k}}{\delta \varphi} \delta_{\theta} \varphi+\frac{\delta \Gamma_{k}}{\delta A} \delta_{\theta} A \\
= & -\frac{1}{2} \operatorname{Tr}\left\{\int d^{d} x\left(\delta_{\theta} \bar{A} \frac{\delta R_{k}}{\delta \bar{A}}\right)\left(\Gamma_{k}^{(2)}+R_{k}\right)^{-1}\right\} \\
& -\frac{1}{\alpha} \int d^{d} x\left(\delta_{\theta} \bar{A}_{\mu}\right) \partial^{\mu} \partial_{\nu} \bar{a}^{\nu} \\
\delta_{\theta}^{\prime}\left(\Gamma_{k}-\Gamma_{\mathrm{gf}}\right)= & -\frac{1}{2} \operatorname{Tr}\left\{\left(\delta_{\theta} R_{k}\right)\left(\Gamma_{k}^{(2)}+R_{k}\right)^{-1}\right\}
\end{aligned}
$$

In the limit where $\Gamma_{k}[\varphi, A, \bar{A}]-\Gamma_{\mathrm{gf}}[A, \bar{A}]$ becomes independent of $\bar{A}$ it also becomes invariant under gauge transformations acting only on $\varphi$ and $A$, and vice versa. With the transformation

$$
\delta_{\theta} \psi=i \theta T \dot{\psi}
$$

one finds

$$
\delta_{\theta} R_{k}=i\left[\theta T, R_{k}\right]
$$


where the generator $T$ is the abelian charge and commutes with $R_{k}[\bar{A}]$. In consequence $\Gamma_{k}[\varphi, A, 0]$ transforms under transformations acting only on $\varphi$ and $A$ as

$$
\begin{aligned}
\delta_{\theta}^{\prime} \Gamma_{k}[\varphi, A, 0]= & -\frac{i}{2} \operatorname{Tr}\left\{T\left[\theta, R_{k}\left(-\partial^{2}\right)\right]\left(\Gamma_{k}^{(2)}[\varphi, A, 0]+R_{k}\left(-\partial^{2}\right)\right)^{-1}\right\} \\
& +\frac{1}{\bar{e} \alpha} \int d^{d} x\left(\partial^{2} \delta \theta\right) \partial_{\nu} A^{\nu}
\end{aligned}
$$

It is instructive to rewrite this transformation in momentum space. With

$$
\theta(x)=\sum_{q} \exp \left(-i q_{\mu} x^{\mu}\right) \theta(q)
$$

the transformation (3.9) is represented by a matrix $\hat{\theta}$,

$$
\begin{aligned}
& \delta_{\theta} \psi(q)=i \hat{\theta}\left(q, q^{\prime}\right) T \psi\left(q^{\prime}\right) \\
& \hat{\theta}\left(q, q^{\prime}\right)=\sum_{Q} \theta(Q) \delta_{q, q^{\prime}+Q}
\end{aligned}
$$

and in eq. (3.11) we have

$$
\left[\hat{\theta}, R_{k}\right]\left(q, q^{\prime}\right)=\sum_{Q} \theta(Q)\left[R_{k}\left((q-Q)^{2}\right)-R_{k}\left(q^{2}\right)\right] \delta_{q, q^{\prime}+Q}
$$

Obviously $\Gamma_{k}[\varphi, A, 0]$ is invariant under global gauge transformations $(Q=0)$. In addition $\Gamma_{k}[\varphi, A, 0]-\Gamma_{\mathrm{gf}}[A, 0]$ becomes locally gauge-invariant if $R_{k}$ is chosen independent of $q^{2}$, for example $R_{k}=Z(k) k^{2}$ with some appropriate $k$-dependent wave function renormalization $Z(k)$. For such a choice $\Delta_{k} S(2.3)$ is manifestly gaugeinvariant since $\chi$ and $a_{\mu}$ transform homogeneously. (We note that $R_{k}$ acts in this case like an additional mass term for the fluctuations.) If $R_{k}$ decays exponentially for $q^{2} \gg k^{2}$, the gauge dependence of $\Gamma_{k}[\varphi, A, 0]-\Gamma_{\mathrm{gf}}[A, 0]$ is also exponentially suppressed for the high momentum modes.

We finally observe that $\left[\theta T, R_{k}\right]$ is antisymmetric in momentum space. The trace in (3.11) therefore vanishes if $\Gamma_{k}^{(2)}$ is diagonal in momentum space. This property can be used to establish the gauge invariance of $\Gamma_{k}[\varphi, A, 0]-\Gamma_{\mathrm{gf}}[A, 0]$ for certain subsets of configurations $(\varphi, A)$.

iv) It follows from (4.7) that $\hat{\Gamma}_{k}^{\text {gauge }}$ also vanishes for $k \rightarrow \infty$. We expect that $\hat{\Gamma}_{k}^{\text {gauge }}$ plays only a role for the modes with eigenvalues of $\mathcal{D}_{\mathrm{S}}$ or $-\partial^{2}$ of the order of $k^{2}$ and reflects, in a sense, a "cutoff effect" required by the gauge-invariant formulation of the infrared cutoff. We do not expect that it plays any major role in the abelian theory. As long as we want to deal with an exact evolution equation, however, the presence of $\hat{\Gamma}_{k}^{\text {gauge }}$ is cumbersome since it contains a large number of possible additional invariants for the formulation with two gauge fields $A$ and $\bar{A}$.

At this point two different strategies may be envisaged. The first evaluates $\Gamma_{k}$ for a fixed background field $\bar{A}$. This strategy becomes particularly simple for the choice $\bar{A}=0$, where one obtains the gauge-fixed exact evolution equation [15] as a straightforward application of the formalism of ref. [9]. In this case one defines the functional

$$
\Gamma_{k}^{\mathrm{b}}[\varphi, A] \equiv \Gamma_{k}[\varphi, A, 0]
$$


(where fb stands for fixed background). It obeys the simple exact evolution equation

$$
\frac{\partial}{\partial t} \Gamma_{k}^{\mathrm{fb}}[\varphi, A]=\frac{1}{2} \operatorname{Tr}\left\{\frac{\partial}{\partial t} R_{k}\left(\Gamma_{k}^{\mathrm{fb}(2)}[\varphi, A]+R_{k}\right)^{-1}\right\}
$$

where $R_{k}$ is now a function of $-\partial^{2}$ and $\Gamma_{k}^{f b(2)}$ is the second functional derivative of $\Gamma_{k}^{\mathrm{fb}}[\varphi, A]$. All methods known from non-gauge theories can be directly employed, as for example a momentum space representation where $R_{k}$ becomes simply a function of $q^{2}$. In particular, this definition is well adapted to small gauge fields $A$ where $\Gamma_{k}^{\mathrm{fb}}$ can be expanded in powers of $A$.

The disadvantage of this method is the lack of gauge invariance for a momentumdependent $R_{k}$. Only the choice $R_{k}=Z(k) k^{2}$ guarantees gauge invariance of $\Gamma_{k}^{\mathrm{fb}}[\varphi, A]$ $-\Gamma_{\mathrm{gf}}[A, 0]$. This choice works presumably rather well in less than four dimensions. Here the evolution equation for the derivatives of $\Gamma_{k}^{\mathrm{fb}}$ with respect to the fields (obtained by differentiating (3.16)) is ultraviolet finite. In four dimensions, however, the derivative of $(3.16)$ with respect to $\varphi$ remains logarithmically divergent for high momenta. This means that in this case the choice $R_{k}=Z(k) k^{2}$ is not sufficient to guarantee that all momentum modes with $q^{2} \gg k^{2}$ are properly integrated out as far as quantities like the scalar mass term in $\Gamma_{k}$ are concerned. An exponential fall off of $R_{k}$, as used in the present paper, remedies this problem. The lack of gauge invariance of $\Gamma_{k}^{\mathrm{fb}}$ does not mean that $\Gamma_{k}^{\mathfrak{h b}}$ contains now more degrees of freedom. In fact, the restrictions due to gauge invariance are now replaced by eq. (3.11).

The second strategy attempts to identify $\bar{A}$ with $A$. This has the advantage that gauge invariance is manifest at every step. Also truncations of the evolution equations which maintain gauge invariance can be easily conceived. As mentioned in the last section, the problem of this strategy stems from the fact that $\Gamma_{k}^{(2)}[\varphi, A, A]$ is not equal to the second functional derivative of $\Gamma_{k}[\varphi, A, A]$ with respect to $\varphi$ and $A$. One part of this difference, related to $\Gamma_{\mathrm{gf}}$, is explicitly known for the abelian theory. The remaining difference which arises from the second functional derivative of $\hat{\Gamma}_{k}^{\text {gauge }}$, may be partly absorbed into a redefinition of $\Gamma_{k}$. We choose a "normalization factor" $\exp -C_{k}[A]$ according to

$$
\begin{aligned}
\Gamma_{k}[\varphi, A] & =\tilde{\Gamma}_{k}[\varphi, A]+C_{k}[A] \\
& =\Gamma_{k}[\varphi, A, A]+C_{k}[A]
\end{aligned}
$$

Here $C_{k}[A]$ is a gauge-invariant functional of $A_{\mu}$ which vanishes for $k \rightarrow 0$ and for $k \rightarrow \infty$. Its second functional derivative should play the role of absorbing the dominant part of the second functional derivative of $\hat{\Gamma}_{k}^{\text {gauge }}$.

We may use (3.2) in order to establish the relations

$$
\begin{aligned}
& \frac{\delta}{\delta A_{\mu}} \Gamma_{k}[\varphi, A, A]=\frac{\delta}{\delta A_{\mu}} \Gamma_{k}[\varphi, A, \bar{A}]_{\mid \bar{A}=A} \\
&+\frac{1}{2} \operatorname{Tr}\left\{\left(\frac{\delta R_{k}[A]}{\delta A_{\mu}}\right)\left(\Gamma_{k}^{(2)}[\varphi, A, A]+R_{k}[A]\right)^{-1}\right\} \\
& \frac{\delta^{2} \Gamma_{k}[\varphi, A, A]}{\delta A_{\mu} \delta A_{\nu}}=\frac{\delta^{2}}{\delta A_{\mu} \delta A_{\nu}} \Gamma_{k}[\hat{H}, A . \bar{A}]_{\mid, \bar{A}=A}+\frac{\delta}{\delta A_{\mu}} \frac{\delta}{\delta \bar{A}_{\nu}} \Gamma_{k}[\varphi, A, \bar{A}]_{\mid \bar{A}=A}
\end{aligned}
$$




$$
\begin{aligned}
+ & \frac{\delta}{\delta \bar{A}_{\mu}} \frac{\delta}{\delta A_{\nu}} \Gamma_{k}[\varphi, A, \bar{A}]_{\mid \bar{A}=A}+\frac{\delta}{\delta \bar{A}_{\mu}} \frac{\delta}{\delta \bar{A}_{\nu}} \Gamma_{k}[\varphi, A, \bar{A}]_{\mid \bar{A}=A} \\
= & \frac{\delta^{2}}{\delta A_{\mu} \delta A_{\nu}} \Gamma_{k}[\varphi, A, \bar{A}]_{\mid \bar{A}=A} \\
& +\frac{1}{2} \operatorname{Tr}\left\{\frac{\delta}{\delta A_{\mu}}\left[\frac{\delta R_{k}[A]}{\delta A_{\nu}}\left(\Gamma_{k}^{(2)}[\varphi, A, A]+R_{k}(A)\right)^{-1}\right]\right\} \\
& -\frac{1}{2} \operatorname{Tr}\left\{\frac{\delta R_{k}[A]}{\delta A_{\mu}}\left(\Gamma_{k}^{(2)}[\varphi, A, A]+R_{k}[A]\right)^{-1} \frac{\delta \Gamma_{k}^{(2)}[\varphi, A, \bar{A}]}{\delta A_{\nu}} \mid \bar{A}=A\right. \\
& \left.\left(\Gamma_{k}^{(2)}[\varphi, A, A]+R_{k}[A]\right)^{-1}\right\}
\end{aligned}
$$

These relations, together with (3.17), can be used to relate the quantity $\Gamma_{k}^{(2)}[\varphi, A, A]$ wich appears in the evolution equation (2.15) to the second functional derivative of $\Gamma_{k}[\varphi, A]$, which we will denote $\Gamma_{k}^{(2)}[\varphi, A]$. Let us write (cf. (3.4))

$$
\Gamma_{k}^{(2)}[\varphi, A, A]=\Gamma_{k}^{(2)}[\varphi, A]+\Gamma_{\mathrm{gf}}^{(2)}+Q_{k}[\varphi, A]
$$

and denote by $Q_{\varphi \varphi}, Q_{\varphi A}, Q_{A \varphi}$ and $Q_{A A}$ the components of the matrix $Q_{k}$ in the various field directions in an obvious notation. Here $\Gamma_{\mathrm{gf}}^{(2)}$ has only a nonvanishing component in the $(A, A)$ direction which is field-independent (cf. (3.3)). One obtains

$$
Q_{k}=\hat{\Gamma}_{k}^{\text {gauge(2) }}-C_{k}^{(2)}
$$

whose matrix elements are given by

$$
\begin{gathered}
Q_{\varphi \varphi}=0 \\
Q_{\varphi_{a} A_{\mu}}=Q_{A_{\mu} \varphi_{a}}=\frac{1}{2} \operatorname{Tr}\left\{\frac{\delta R_{k}[A]}{\delta A_{\mu}}\left(\Gamma_{k}^{(2)}[\varphi, A]+\Gamma_{\mathrm{gf}}^{(2)}+Q_{k}[\varphi, A]+R_{k}[A]\right)^{-1}\right. \\
\frac{\delta}{\delta \varphi_{a}}\left(\Gamma_{k}^{(2)}[\varphi, A]+Q_{2}[\varphi, A]\right) \\
\left.\cdot\left(\Gamma_{k}^{(2)}[\varphi, A]+\Gamma_{\mathrm{gf}}^{(2)}+Q_{k}[\varphi, A]+R_{k}[A]\right)^{-1}\right\} \\
\left.Q_{A_{\mu} A_{\nu}=-\frac{\delta^{2} C_{k}[A]}{\delta A_{\mu} \delta A_{\nu}}}\right\} \\
-\frac{1}{2} \operatorname{Tr}\left\{\frac{\delta}{\delta A_{\mu}}\left[\frac{\delta R_{k}[A]}{\delta A_{\mu}}\left(\Gamma_{k}^{(2)}[\varphi, A]+\Gamma_{\mathrm{gf}}^{(2)}+Q_{k}[\varphi, A]+R_{k}[A]\right)^{-1}\right]\right\} \\
+\frac{1}{2} \operatorname{Tr}\left\{\frac{\delta R_{k}[A]}{\delta A_{\mu}}\left(\Gamma_{k}^{(2)}[\varphi, A]+\Gamma_{\mathrm{gf}}^{(2)}+Q_{k}[\varphi, A]+R_{k}[A]\right)^{-1} \frac{\delta}{\delta A_{\nu}}\left(\Gamma_{k}^{(2)}[\varphi, A]+Q_{k}[\varphi, A]\right)\right.
\end{gathered}
$$

${ }^{4}$ Note that $\Gamma_{k}^{(2)}[\varphi, A] \neq \Gamma_{k}^{(2)}[\varphi, A, A]$. Whenever a confusion is possible, we therefore write explicitly the arguments in order to distinguish these quantities. 


$$
\begin{aligned}
& \left.\left(\Gamma_{k}^{(2)}[\varphi, A]+\Gamma_{\mathrm{gf}}^{(2)}+Q_{k}[\varphi, A]+R_{k}[A]\right)^{-1}\right\} \\
& -\frac{1}{2} \operatorname{Tr}\left\{\frac{\delta R_{k}[A]}{\delta A_{\mu}}\left(\Gamma_{k}^{(2)}[\varphi, A]+\Gamma_{\mathrm{gf}}^{(2)}+Q_{k}[\varphi, A]+R_{k}[A]\right)^{-1} \frac{\delta \Gamma_{k}^{(2)}[\varphi, A, \bar{A}]}{\delta \bar{A}_{\nu}} \mid \bar{A}=A\right. \\
& \left.\left(\Gamma_{k}^{(2)}[\varphi, A]+\Gamma_{\mathrm{gf}}^{(2)}+Q_{k}[\varphi, A]+R_{k}[A]\right)^{-1}\right\}
\end{aligned}
$$

An exact solution of $Q_{k}$ in terms of $\Gamma_{k}$ seems extremely difficult. One may, however, employ an iterative procedure if $Q_{k}[\varphi, A]$ is small compared to $\Gamma_{k}^{(2)}[\varphi, A]+\Gamma_{\mathrm{gf}}^{(2)}+R_{k}[A]$ in the region which dominates the momentum integration implied by the trace (i.e. $q^{2} \approx k^{2}$ ). Also the derivatives of $Q_{k}$ with respect to $\varphi$ and $A$ should be small compared to the corresponding derivatives of $\Gamma_{k}^{(2)}[\varphi, A]$. One can then neglect $Q_{k}$ on the r.h.s. of (3.23) and (3.24) in a first approximation, and, if necessary, compute corrections by inserting the lower order approximations of $Q_{k}$ in consecutive steps. A particular problem arises from the factor $\delta \Gamma_{k}^{(2)}[\varphi, A, \bar{A}] / \delta \bar{A}_{\nu \mid \bar{A}=A}$ in the last term in (3.24). This quantity has no direct expression in terms of $\Gamma_{k}$ and $Q_{k}$ and can only be evaluated iteratively by taking the second functional derivative of $(3.2)$ with respect to $\varphi$ and $A$ at fixed $\bar{A}$. It is clear that in practice this second startegy will be useful only if the corrections due to $Q_{k}$ are small. This works best for a smooth function $R_{k}$ since $Q_{k}$ vanishes identically for $R_{k}=$ const. In addition, the optimization of this strategy depends on the choice of $C_{k}$ in (3.17) since $C_{k}^{(2)}$ can be used to cancel leading order effects on the r.h.s. of (3.24).

In the present paper we concentrate on the second strategy. In the next sections we give in this formulation the exact evolution equation. We compute explicitly the scale dependence of the gauge coupling, the scalar potential and wave function renormalization in an appropriate truncation. This should give a reliable nonperturbative description of the abelian Higgs model in arbitrary dimensions.

\section{Gauge-invariant evolution equation}

We can now write down the exact evolution equation for the gauge-invariant effective action $\Gamma_{k}[\varphi, A]$ of eq. (3.17) for the abelian theory:

$$
\begin{aligned}
\frac{\partial}{\partial t} \Gamma_{k}[\varphi, A] & =\frac{\partial}{\partial t} C_{k}[A] \\
& +\frac{1}{2} \operatorname{Tr}\left\{\left(\frac{\partial}{\partial t} R_{k}[A]\right)\left(\Gamma_{k}^{(2)}[\hat{\varphi}, A]+\Gamma_{\mathrm{gf}}^{(2)}+Q_{k}[\varphi, A]+R_{k}[A]\right)^{-1}\right\}
\end{aligned}
$$

It expresses the $k$-dependence of $\Gamma_{k}$ in terms of its second functional derivative $\Gamma_{k}^{(2)}$ and corresponds to eq. (1.4) in the introduction. The correction matrix $Q_{k}$ is given by (3.22)-(3.24) and depends on the choice of the normalization factor $C_{k}$. We expand the evolution equation in powers of $Q_{k}$ :

$$
\frac{\partial}{\partial t} \Gamma_{k}[\varphi, A]=\gamma_{k}^{(0)}[\varphi, A]+\gamma_{k}^{(1)}[\varphi, A]+\ldots
$$




$$
\begin{aligned}
\gamma_{k}^{(0)}[\varphi, A]= & \bar{\gamma}_{k}+\frac{\partial}{\partial t} C_{k}[A] \\
\bar{\gamma}_{k}= & \left.\frac{1}{2} \operatorname{Tr}\left\{\frac{\partial}{\partial t} R_{k}[A]\right)\left(\Gamma_{k}^{(2)}[\varphi, A]+\Gamma_{\mathrm{gf}}^{(2)}+R_{k}[A]\right)^{-1}\right\} \\
\gamma_{k}^{(1)}[\varphi, A]= & -\frac{1}{2} \operatorname{Tr}\left\{\left(\frac{\partial}{\partial t} R_{k}[A]\right)\left(\Gamma_{k}^{(2)}[\varphi, A]+\Gamma_{\mathrm{gf}}^{(2)}+R_{k}[A]\right)^{-1}\right. \\
& \left.\cdot Q_{k}[\varphi, A]\left(\Gamma_{k}^{(2)}[\varphi, A]+\Gamma_{\mathrm{gf}}^{(2)}+R_{k}[A]\right)^{-1}\right\}
\end{aligned}
$$

Let us now discuss the optimal choice for $C_{k}$ which makes $Q_{k}$ as small as possible. We are mainly interested in small and slowly varying gauge fields - typically $F_{\mu \nu} F^{\mu \nu} \ll k^{4}, A_{\mu}^{2} \ll k^{2}$ - and slowly varying scalar fields, i.e. $\left|D_{\mu} \varphi\right|^{2} \ll k^{4}$. From the functional form of $R_{k}$ it is obvious that the traces (3.23) and (3.24), which determine $Q_{k}$, are dominated by a small momentum region $q^{2} \approx k^{2}$. We therefore may approximate the kinetic terms in $\Gamma_{k}^{(2)}[\varphi, A, A]$ by $\mathcal{Z}_{k} k^{2}$ with $\mathcal{Z}_{k}$ on appropriate (diagonal) matrix accounting for wave function renormalization effects. Similarly we introduce a mass matrix $\mathcal{M}_{k}^{2}$ which should be as close as possible to the relevant mass matrix in $\Gamma_{k}^{(2)}[\varphi, A, A]$. This suggests the following definition for the "normalization factor"

$$
C_{k}[A]=-\frac{1}{2} \operatorname{Tr}\left\{\ln \left(R_{k}[A]+\mathcal{Z}_{k} k^{2}+\mathcal{M}_{k}^{2}\right)-\ln \left(\mathcal{Z}_{k} k^{2}+\mathcal{M}_{k}^{2}\right)\right\}
$$

Since $C_{k}$ should be gauge-, translation- and rotation-invariant, the matrix $\mathcal{Z}_{k} k^{2}+\mathcal{M}_{k}^{2}$ must respect these symmetries. In practice this means that $\mathcal{Z}_{k}$ and $\mathcal{M}_{k}^{2}$ have to be diagonal with identical eigenvalues for the two components of a charged scalar field and identical eigenvalues for the $d-1$ components of the transverse gauge field. (More details concerning the choice and properties of $C_{k}[A]$ can be found at the end of this section).

In the limit where $\Gamma_{k}^{(2)}[\varphi, A, A]$ equals $\mathcal{Z}_{k} k^{2}+\mathcal{M}_{k}^{2}$ one finds $Q_{k}=0$. In particular, the first term in (3.24) from $C_{k}^{(2)}$ cancels the second term. To the extent that $\mathcal{Z}_{k} k^{2}+\mathcal{M}_{k}^{2}$ is a good approximation to $\Gamma_{k}^{(2)}$ for $q^{2} \approx k^{2}$, the correction $Q_{k}$ is therefore small ${ }^{5}$, and the expansion (4.2) is justified. The lowest order approximation for $Q_{k}$ reads

$$
\begin{aligned}
Q_{\varphi_{a} A_{\mu}}= & Q_{A_{\mu} \varphi_{a}}=\frac{1}{2} \operatorname{Tr}\left\{\frac{\delta R_{k}[A]}{\delta A_{\mu}}\left(\Gamma_{k}^{(2)}[\vartheta, A]+\Gamma_{\mathrm{gf}}^{(2)}+R_{k}[A]\right)^{-1} \frac{\delta \Gamma_{k}^{(2)}[\varphi, A]}{\delta \varphi_{a}}\right. \\
& \left.\left(\Gamma_{k}^{(2)}[\varphi, A]+\Gamma_{\mathrm{gf}}^{(2)}+R_{k}[A]\right)^{-1}\right\} \\
Q_{A_{\mu} A_{\nu}}= & \frac{1}{2} \operatorname{Tr}\left\{\frac{\delta^{2} R_{k}[A]}{\delta A_{\mu} \delta A_{\nu}}\left[\left(\mathcal{Z}_{k} k^{2}+\mathcal{M}_{k}^{2}+R_{k}[A]\right)^{-1}-\left(\Gamma_{k}^{(2)}[\vartheta, A]+\Gamma_{\mathrm{gf}}^{(2)}+R_{k}[A]\right)^{-1}\right]\right\}
\end{aligned}
$$

\footnotetext{
${ }^{3}$ One could also consider the possibility that $\mathcal{M}_{k}^{2}$ and $\mathcal{Z}_{k}$ depend on invariants formed from $\vec{\gamma}$, e.g. $\rho \equiv \frac{1}{2} \varphi_{a} \varphi^{a}$. Then (4.3) would be generalized to

$$
C_{k}[\rho, A]=-\frac{1}{2} \operatorname{Tr}\left[\ln \left(R_{k}[A]+\mathcal{Z}_{k}(\rho) k^{2}+\mathcal{M}_{k}^{2}(\rho)\right)-\ln \left(R_{k}(0)+\mathcal{Z}_{k}(\rho) k^{2}+\mathcal{M}_{k}^{2}(\rho)\right)\right]
$$
}

and appropriate correction factors appear also in $Q_{9.4}$. 


$$
\begin{aligned}
& -\frac{1}{2} \operatorname{Tr}\left\{\frac { \delta R _ { k } [ A ] } { \delta A _ { \nu } } \left[\left(\mathcal{Z}_{k} k^{2}+\mathcal{M}_{k}^{2}+R_{k}[A]\right)^{-1} \frac{\delta R_{k}[A]}{\delta A_{\mu}}\left(\mathcal{Z}_{k} k^{2}+\mathcal{U}_{k}^{2}+R_{k}[A]\right)^{-1}\right.\right. \\
& \left.\left.-\left(\Gamma_{k}^{(2)}[\varphi, A]+\Gamma_{\mathrm{gf}}^{(2)}+R_{k}[A]\right)^{-1} \frac{\delta R_{k}[A]}{\delta A_{\mu}}\left(\Gamma_{k}^{(2)}[\varphi, A]+\Gamma_{\mathrm{gf}}^{(2)}+R_{k}[A]\right)^{-1}\right]\right\} \\
& +\frac{1}{2} \operatorname{Tr}\left\{\left[\frac{\delta R_{k}[A]}{\delta A_{\mu}}\left(\Gamma_{k}^{(2)}[\varphi, A]+\Gamma_{\mathrm{gf}}^{(2)}+R_{k}[A]\right)^{-1} \frac{\delta \Gamma_{k}^{(2)}[\varphi, A]}{\delta A_{\nu}}\right.\right. \\
& \left.\left.+\frac{\delta R_{k}[A]}{\delta A_{\nu}}\left(\Gamma_{k}^{(2)}[\varphi, A]+\Gamma_{\mathrm{gf}}^{(2)}+R_{k}[A]\right)^{-1} \frac{\delta \Gamma_{k}^{(2)}[\varphi, A]}{\delta A_{\mu}}\right]\left(\Gamma_{k}^{(2)}[\varphi, A]+\Gamma_{\mathrm{gf}}^{(2)}+R_{k}[A]\right)^{-1}\right\}
\end{aligned}
$$

One sees explicitly that a nonvanishing $Q_{k}$ arises only through the field dependence of $\Gamma_{k}^{(2)}$ and its deviation from $\mathcal{Z}_{k} k^{2}+\mathcal{M}_{k}^{2}$.

We emphasize at this place that our arguments on the smallness of $Q_{k}$ are not based on a loop expansion or on an expansion in the small gauge coupling $\bar{e}$. If such expansions are valid, we may in addition classify the order of $Q_{k}$ in this respect. Indeed, $Q_{k}$ is always proportional $\delta R_{k} / \delta A$ and therefore vanishes for $\bar{e} \rightarrow 0$. The trace stands for a loop integral. One concludes that the r.h.s. of the evolution equation (4.1) is of one-loop order. Also $C_{k}$ and its contribution to the evolution equation is formally of one-loop order. In this language $\Gamma_{k}, \Gamma_{k}^{(2)}$ and $R_{k}$ count as zeroth order. In consequence, the one-loop contribution $Q_{k}$ is one order higher than $\Gamma_{k}^{(2)}+R_{k}$ and the expression $\gamma_{k}^{(1)}$ of eq. (4.2) should be considered as a twoloop contribution. The iterative solution of (3.23), (3.24) can also be justified by a loop expansion. In particular, the background field dependence of $\Gamma_{k}$ is of one-loop order according to (3.2). This implies that the last term in (3.24) only contributes in two-loop order and can be neglected in a first approximation as in (4.5). We emphasize, nevertheless, that $\gamma_{k}^{(0)}$ contains much more than a one-loop contribution! The corresponding expression is exact to all loops for the pure scalar theory and should hold with high precision whenever $\Gamma_{k}^{(2)} \approx \mathcal{Z}_{k} k^{2}+\mathcal{M}_{k}^{2}$ for momenta $q^{2} \approx k^{2}$.

We will not evaluate $Q_{k}$ in this paper and restrict our discussion to $\gamma_{k}^{(0)}$. Except for the correction from the normalization factor $C_{k}$ and the contribution $\Gamma_{\mathrm{gf}}^{(2)}$ from the gauge fixing, the evolution equation has then exactly the same form as the exact nonperturbative evolution equation for the pure scalar theory [9]. We observe that $\bar{\gamma}_{k}$ has the form of the $t$-derivative of the standard one-loop expression

$$
\bar{\gamma}_{k}=\frac{1}{2} \operatorname{Tr} \frac{\partial}{\partial t} \ln \left(\Gamma_{k}^{(2)}[\hat{\varphi}, A]+\Gamma_{g f}^{(2)}+R_{k}[A]\right)
$$

(Here $\frac{\partial}{\partial t}$ acts only on $R_{k}$ at fixed $\Gamma_{k}^{(2)}$.) Compared with the standard one-loop expression, there are two important modifications: First, the effective propagator has an additional $k$-dependent piece $R_{k}$ acting as an infrared cutoff. In addition, the $t$-derivative makes the integral ultraviolet finite. The second modification is the appearance of $\Gamma_{k}^{(2)}$ instead of the second functional derivative of the classical action. This "renormalization group improvement" contains the essential part of the higher-loop contributions once the contribution from $C_{k}$ is included. All missing corrections are then contained in $\gamma_{k}^{(1)}$ and higher orders in $Q_{k}$. 
We can also include the contribution from $C_{k}$ into the correction to the field equations. One has

$$
\begin{gathered}
J=\frac{\delta \Gamma[\varphi, A]}{\delta \varphi} \\
K=\frac{\delta \Gamma[\varphi, A]}{\delta A}-\tilde{\kappa}=\frac{\delta \bar{\Gamma}[\varphi, A]}{\delta A}-\kappa
\end{gathered}
$$

This yields

$$
\begin{aligned}
\tilde{\kappa}[\varphi, A]= & \frac{1}{2} \operatorname{Tr}\left\{\frac { \delta R _ { k } ( A ) } { \delta A } \left[\left(\Gamma_{k}^{(2)}[\varphi, A, A]+R_{k}[A]\right)^{-1}\right.\right. \\
& \left.\left.-\left(\mathcal{Z}_{k} k^{2}+\mathcal{M}_{k}^{2}+R_{k}[A]\right)^{-1}\right]\right\}
\end{aligned}
$$

Using these expressions in (4.5) we finally obtain the functional integral representation for the abelian effective average action $\Gamma_{k}[\varphi, A]$ :

$$
\begin{aligned}
& \exp -\Gamma_{k}[\varphi, A]=\exp -C_{k}[A] \int D \chi D a \exp -\left\{S[\varphi+\chi, A+a]+S_{\mathrm{gf}}[a]\right. \\
& \left.+\Delta_{k} S[\chi, a ; A]-\int d^{d} x\left(\frac{\delta \Gamma_{k}[\varphi, A]}{\delta \varphi} \chi+\frac{\delta \Gamma_{k}[\varphi, A]}{\delta A} a-\tilde{\kappa}[\varphi, A] a\right)\right\}
\end{aligned}
$$

This is the gauge-invariant effective action (1.3) proposed in the introduction. The gauge-invariant normalization factor

$$
\exp -C_{k}[A]=\operatorname{det}\left[\frac{R_{k}[A]+\mathcal{Z}_{k} k^{2}+\mathcal{M}_{k}^{2}}{\mathcal{Z}_{k} k^{2}+\mathcal{M}_{k}^{2}}\right]^{1 / 2}
$$

fulfils exactly the role of the normalization factor $\operatorname{det}_{<}[H]$ discussed earlier in ref. [8].

The normalization factor contributes to the scale dependence of $\Gamma_{k}$ according to

$$
\begin{aligned}
\frac{\partial}{\partial t} C_{k}[A]= & -\frac{1}{2} \operatorname{Tr}\left\{\left(\frac{\partial}{\partial t} R_{k}[A]+\left(2+\frac{\partial}{\partial t} \ln \mathcal{Z}_{k}\right) \mathcal{Z}_{k} k^{2}+\frac{\partial}{\partial t} \mathcal{M}_{k}^{2}\right)\right. \\
& \cdot\left(R_{k}[A]+\mathcal{Z}_{k} k^{2}+\mathcal{M}_{k}^{2}\right)^{-1} \\
& \left.-\left(\left(2+\frac{\partial}{\partial t} \ln \mathcal{Z}_{k}\right) \mathcal{Z}_{k} k^{2}+\frac{\partial}{\partial t} \mathcal{M}_{k}^{2}\right)\left(\mathcal{Z}_{k} k^{2}+\mathcal{M}_{k}^{2}\right)^{-1}\right\}
\end{aligned}
$$

Since $C_{k}$ is gauge-invariant, we may expand

$$
C_{k}[A]=\int d^{d} x \frac{1}{4} Z_{c, k} F_{\mu \nu} F^{\mu \nu}+\ldots
$$

and extract the $k$-dependence of $Z_{i}$ from the term quadratic in $A_{\mu}$ on the r.h.s. of eq. (4.12). A technically more convenient method is to evaluate $C_{k}[A]$ for a gauge field corresponding to a constant magnetic field $B$ and to read off $Z_{c, k}$ from the term proportional to $B^{2}$. (This calculation is similar to the one in ref. [8].) One obtains 
in the symmetric phase of the abelian Higgs model, with $m_{k}^{2}$ the renormalized $k$ dependent scalar mass term and $Z_{\varphi, k}$ the scalar wave function renormalization

$$
\begin{aligned}
& \frac{d}{d t} Z_{c, k}=-\lim _{B \rightarrow 0}\left\{\frac{2}{B^{2}} \operatorname{Tr} \frac{d}{d t} \ln \left[1+\frac{R_{k}^{(\mathcal{S})}\left(-D^{2}(B)\right)}{Z_{\varphi, k}\left(k^{2}+m_{k}^{2}\right)}\right]\right\} \\
& =-v_{d-2} \frac{\bar{e}^{2}}{6 \pi} \int_{0}^{\infty} d x x^{\frac{d-4}{2}} \frac{d}{d x} \frac{d}{d t} \ln \left(1+\frac{R_{k}^{(S)}(x)}{Z_{\varphi, k}\left(k^{2}+m_{k}^{2}\right)}\right)
\end{aligned}
$$

Here the trace in (4.14) acts only in scalar space and includes a summation over momenta with $q^{2}=x$ with

$$
v_{d}^{-1}=2^{d+1} \pi^{\frac{d}{2}} \Gamma\left(\frac{d}{2}\right)
$$

The last equation in (4.14) applies only for $d>2$. In particular, one finds for $d=4$ :

$$
\frac{d}{d t} Z_{c, k}=\frac{\bar{e}^{2}}{48 \pi^{2}} \lim _{x \rightarrow 0} \frac{d}{d t} \ln \frac{R_{k}^{(S)}(x)+Z_{\varphi, k}\left(k^{2}+m_{k}^{2}\right)}{Z_{\varphi, k}\left(k^{2}+m_{k}^{2}\right)}
$$

For $m_{k}^{2}>0$ the definition (2.5) yields

$$
\frac{d}{d t} Z_{c, k}=\frac{\bar{e}^{2}}{48 \pi^{2}} \frac{d}{d t} \ln \left(1+\frac{f_{k}^{2}\left(m_{k}^{2}\right) m_{k}^{2}}{\left(1-f_{k}^{2}\left(m_{k}^{2}\right)\right)\left(k^{2}+m_{k}^{2}\right)}\right)
$$

This contribution is exponentially suppressed for $k^{2} \ll m_{k}^{2}$ and also very small for $k^{2} \gg m_{k}^{2}$ if we employ $b=1$ in eq. (2.6). For the regime with spontaneous symmetry breaking we use $m_{k}^{2}=0$. In this case it is instructive to compare the equations for $\frac{d}{d t} Z_{c, k}$ resulting for cut-off functions $f_{k}\left(q^{2}\right)(2.6)$ with $b>1$ and $b=1$, respectively. For $b>1, R_{k}(x)$ diverges $\sim k^{2 b} x^{1-b} Z_{\varphi, k}$ for $x \rightarrow 0$. In this case eq. (4.16) leads to

$$
\frac{d}{d t} Z_{c, k}^{(b>1)}=\frac{e^{2}}{24 \pi^{2}}(b-1)
$$

For $b=1, R_{k}(x)$ remains finite for $x \rightarrow 0$. Our normalization $R_{k}^{(\mathcal{S})}(0)=Z_{\varphi, k} k^{2}$ (2.5) entails

$$
\frac{d}{d t} Z_{c, k}^{(b=1)}=0
$$

As stated before, we will concentrate in this paper on $b=1$.

\section{Approximate evolution equations for the abelian Higgs model}

Neglecting the small corrections $\sim Q_{k}$ discussed in the last section, the evolution equation describes the scale dependence of the gauge-invariant average action $\Gamma_{k}[\varphi, A]$ in terms of its second functional derivative

$$
\frac{\partial}{\partial t} \Gamma_{k}[\varphi, A]=\frac{1}{2} \operatorname{Tr}\left\{\frac{\partial \hat{R}_{k}[A]}{\partial t}\left(\Gamma_{k}^{(2)}[\varphi, A]+\hat{R}_{k}[A]\right)^{-1}\right\}+\frac{\partial}{\partial t} C_{k}[A]
$$


Here we have absorbed the simple contribution from the gauge-fixing term in the definition $\hat{R}_{k}=R_{k}+S_{\mathrm{gf}}^{(2)}$. (This only adds to $\tilde{R}_{k}^{(G)}$ in (2.t) in a term $-\partial^{2}$.) This equation remains a complicated coupled nonlinear differential equation for the infinitely many couplings characterizing $\Gamma_{k}[\varphi, A]$. Any use of this equation has to proceed to some truncation. We will demonstrate this here for the abelian Higgs model. It is defined by the classical action

$$
S=\int d^{d} x\left\{\frac{1}{4} F_{\mu \nu} F^{\mu \nu}+\left|D_{\mu} \varphi\right|^{2}-\bar{\mu}^{2}|\varphi|^{2}+\frac{1}{2} \bar{\lambda}|\varphi|^{4}\right\}
$$

where $D_{\mu} \equiv \partial_{\mu}+i \bar{e} A_{\mu}$, and $\bar{e}, \bar{\lambda}$ and $\bar{\mu}$ are the bare parameters of the theory. We expand $\left(\rho \equiv \varphi^{*} \varphi\right)$

$$
\begin{aligned}
\Gamma_{k}[\varphi, A]=\int d^{d} x\left\{U_{k}(\rho)\right. & +Z_{\varphi, k}(\rho)\left(D_{\mu} \varphi\right)^{*} D^{\mu} \varphi \\
& \left.+\frac{1}{4} Z_{F, k}(\rho) F_{\mu \nu} F^{\mu \nu}+\ldots\right\}
\end{aligned}
$$

and neglect the higher derivative terms. Furthermore, we shall restrict the dependence on $\rho$ of the functions $U_{k}, Z_{\varphi, k}$ and $Z_{F, k}$. Let. $\rho_{0}$ denote the location of the minimum of the potential $U_{k}$. In the symmetric regime one has $\rho_{0}=0$ whereas in the regime with spontaneous symmetry breaking the minimum occurs for nonvanishing $\rho_{0}(k)$ with $U_{k}^{\prime}\left(\rho_{0}\right)=0$. (The prime denotes differentiation with respect to $\rho$.) We keep here at most second derivatives $U^{\prime \prime}\left(\rho_{0}\right)$, but discard higher terms such as $U_{k}^{\prime \prime \prime}\left(\rho_{0}\right)$. We also approximate the wave function renormalizations by their values at the minimum of $U_{k}: Z_{\varphi, k} \equiv Z_{\varphi, k}\left(\rho_{0}\right), Z_{F, k} \equiv Z_{F, k}\left(\rho_{0}\right)$.

Concerning the choice of the infrared cutoff (2.5), we employ in this section $m_{k}^{2}=M_{k}^{2}=0, Z_{G, k}=1$. This choice allows to combine the kinetic terms in $\Gamma_{k}$ and the infrared constraint in a simple way

$$
P(x)=x+\frac{x f_{k}^{2}(x)}{1-f_{k}^{2}(x)}=\frac{x}{1-f_{k}^{2}(x)}
$$

The second variation of the truncated form of $\Gamma_{k}$, together with our choice of $R_{k}$, leads then to the quadratic form

$$
\begin{aligned}
& \frac{1}{2} \int d^{d} x \delta \psi\left[\Gamma_{k}^{(2)}+\hat{R}_{k}\right] \delta \psi \\
& =\frac{1}{2} \int d^{d} x\left\{\delta A _ { \mu } \left[Z_{F, k} P\left(-\partial^{2}\right)\left(\delta^{\mu \nu}-\frac{\partial^{\mu} \partial^{\nu}}{\partial^{2}}\right)+\frac{1}{\alpha} P\left(-\partial^{2}\right) \frac{\partial^{\mu} \partial^{\nu}}{\partial^{2}}\right.\right. \\
& \left.+2 Z_{\varphi, k} \bar{e}^{2} \rho \delta^{\mu \nu}\right] \delta A_{\nu} \\
& +2 \delta \varphi^{*}\left[Z_{\varphi, k} P\left(-D^{2}\right)+U_{k}^{\prime}(\rho)+\rho U_{k}^{\prime \prime}(\rho)\right] \delta \varphi \\
& +U_{k}^{\prime \prime}(\rho)\left[\varphi^{2}\left(\delta \varphi^{*}\right)^{2}+\varphi^{* 2}(\delta \varphi)^{2}\right] \\
& +4 Z_{\varphi, k} \bar{e}^{-2} \delta A_{\mu} A^{\mu}\left(\varphi \delta \varphi^{*}+\varphi^{*} \delta \varphi\right) \\
& \left.-2 i Z_{\varphi, k} \bar{e} \delta A_{\mu}\left[\delta \varphi^{*} \partial^{\mu} \varphi-\varphi \partial^{\mu} \delta \varphi^{*}+\hat{\varphi}^{*} \partial^{\mu} \delta \varphi-\delta \varphi \partial^{\mu} \varphi^{*}\right]\right\}
\end{aligned}
$$


This is a quadratic action for the fluctuations $\delta \psi \equiv\left(\delta A_{\mu}, \delta \varphi, \delta \varphi^{*}\right)$. It defines the operator $\Gamma_{k}^{(2)}+\hat{R}_{k}$ which enters $(j .1)$. In order to evaluate the trace on the r.h.s. of eq. (5.1), we note that we may formally rewrite it as

$$
\frac{\partial}{\partial t} \Gamma_{k}[\varphi, A]=\frac{\partial}{\partial t} C_{k}[A]+\frac{1}{2} \frac{\partial}{\partial t} \ln \operatorname{det}\left[\Gamma_{k}^{(2)}+\hat{R}_{k}\right]
$$

whereby the partial derivative $\frac{\partial}{\partial t}$ acts on the r.h.s. of (5.6) only on the explicit $k$-dependence of $R_{k}$, but not on $\Gamma_{k}^{(2)}$. The determinant in (5.6) is closely related to the one which appears in the one-loop approximation of the average action [7],[8]. In fact, eq. (5.6) amounts to a renormalization-group improved one-loop calculation: instead of the second variation of the classical action $S^{(2)}$, the determinant contains the field- and scale-dependent inverse propagator $\Gamma_{k}^{(2)}+\hat{R}_{k}$. The average action of the abelian Higgs model was introduced and analysed in refs. [7], and [8]. In these papers we described in detail the evaluation of $\partial_{t} \ln \operatorname{det}\left[S^{(2)}+\hat{R}_{k}\right]$. We can take advantage of the results obtained there if we note the following changes. Clearly we have to replace $S^{(2)}$ by $\Gamma_{k}^{(2)}$ which, in view of the truncations made here, amounts to replacing the bare parameters $-\bar{\mu}^{2}$ and $\bar{\lambda}$ by $U_{k}^{\prime}-\rho U_{k}^{\prime \prime}$ and $U_{k}^{\prime \prime}$, respectively. Furthermore, $A_{\mu}$ and $\varphi$ have to be multiplied by appropriate $Z$-factors ${ }^{6}$. The most important simplification concerns the form of the cut-off function $f_{k}\left(q^{2}\right)$. In [7] and [8] we were forced to use values $b>2$, now we shall set $b=1$ and $a=\frac{1}{2}$ in (2.6). With the present truncation the flow equations for the scalar potential and $Z_{\varphi, k}$ are exactly the same as those derived in ref. [7]. We give here only the results for the value $\alpha=0$ of the gauge-fixing parameter. The corresponding results for general values of $\alpha$ can be found in ref. [7] to which the reader is referred also for further calculational details. We also neglect in a first approximation the $k$-dependence of $Z_{\varphi, k}$ and $Z_{F, k}$ in $\partial R_{k} / \partial t$.

We begin with the results for the theory in the regime with spontaneous symmetry breaking. The renormalization group equations are most conveniently expressed in terms of the dimensionless quantities

$$
\begin{aligned}
\kappa(k) & =k^{2-d} Z_{\varphi, k} \rho_{0}(k) \\
\lambda(k) & =k^{d-4} Z_{-. k}^{-2} U_{k}^{\prime \prime}\left(\rho_{0}(k)\right) \\
e^{2}(k) & =k^{d-4} Z_{F, k}^{-1} \bar{e}^{2}
\end{aligned}
$$

Here $\kappa$ is the renormalized and dimensionless "running minimum" of the potential $U_{k}(\rho)$. In terms of the above quantities the running gauge boson mass and radial scalar mass, for example, are given by $M_{k}^{2}=2 e^{2} \kappa k^{2}$ and $m_{r, k}^{2} \equiv 2 \lambda \kappa k^{2}$. respectively. For $k \rightarrow 0$ they give the physical mass term (defined at zero momentum rather than at the pole). We also introduce the anomalous dimensions

$$
\begin{aligned}
& \eta_{\varphi}=-\frac{\partial}{\partial t} \ln Z_{\vartheta, k} \\
& \eta_{F}=-\frac{\partial}{\partial t} \ln Z_{F, k}
\end{aligned}
$$

\footnotetext{
${ }^{6}$ They can be deduced by comparing (5.5) to the analogous equation( $(3.4)$ in ref. $[7]$.
} 
For the running of $\kappa$ and $\lambda$ one finds for the approximation of a quartic potential

$$
\begin{aligned}
& \frac{\partial}{\partial t} \kappa=\left(2-d-\eta_{\varphi}\right) \kappa+2 v_{d} l_{1}^{d}+6 v_{d} l_{1}^{d} s_{1}^{d}(2 \lambda \kappa) \\
&+4(d-1) v_{d} \frac{e^{2}}{\lambda} l_{1}^{d} s_{1}^{d}\left(2 e^{2} \kappa\right) \\
& \frac{\partial}{\partial t} \lambda=\left(d-4+2 \eta_{\varphi}\right) \lambda+2 v_{d} l_{2}^{d} \lambda^{2}+18 v_{d} \lambda^{2} l_{2}^{d} s_{2}^{d}(2 \lambda \kappa) \\
&+8(d-1) v_{d} e^{4} l_{2}^{d} s_{2}^{d}\left(2 e^{2} \kappa\right)
\end{aligned}
$$

Here the "threshold functions" $s_{n}^{d}$ and the constants $l_{n}^{d}$

$$
s_{n}^{d}(w) \equiv l_{n}^{d}(w) / l_{n}^{d}(0), \quad l_{n}^{d} \equiv l_{n}^{d}(0)
$$

are defined in terms of the dimensionless integrals $[4]$

$$
l_{n}^{d}(w)=-\frac{1}{2} k^{2 n-d} \int_{0}^{\infty} d x x^{d / 2-1} \frac{\partial}{\partial t}\left[P(x)+w k^{2}\right]^{-n}
$$

In particular, one recovers for $d=4$ and $w=0$ the one-loop perturbative result since $l_{2}^{4}=1$. The threshold functions $s_{n}^{d}(w)$ describe how the running of the various quantities changes once $k$ drops below some physical mass scale $w k$ which acts as an infrared cutoff. The functions $s_{n}^{d}(w)$ vanish for $w \gg 1$ and they approach unity for $w \ll 1$. The anomalous dimension of the scalar is given by

$$
\begin{aligned}
\eta_{\varphi}= & -16 v_{d}\left(1-d^{-1}\right) e^{2} l_{1,1}^{d}\left(2 e^{2} \kappa, 2 \lambda \kappa\right) \\
& +16 d^{-1} v_{d} \lambda^{2} \kappa m_{2,2}^{d}(2 \lambda \kappa, 0)
\end{aligned}
$$

It involves the integrals

$$
\begin{aligned}
l_{n_{1}, n_{2}}^{d}\left(w_{1}, w_{2}\right)= & -\frac{1}{2} k^{2\left(n_{1}+n_{2}\right)-d} \int_{0}^{\infty} d x x^{d / 2-1} \\
& \cdot \frac{\partial}{\partial t}\left[\left(P(x)+w_{1} k^{2}\right)^{-n_{1}}\left(P(x)+w_{2} k^{2}\right)^{-n_{2}}\right] \\
m_{n_{1}, n_{2}}^{d}\left(w_{1}, w_{2}\right)= & -\frac{1}{2} k^{2\left(n_{1}+n_{2}-1\right)-d} \int_{0}^{\infty} d x x^{d / 2} . \\
& \cdot \frac{\partial}{\partial t}\left[\left(\frac{d P}{d x}\right)^{2}\left(P(x)+w_{1} k^{2}\right)^{-n_{1}}\left(P(x)+w_{2} k^{2}\right)^{-n_{2}}\right]
\end{aligned}
$$

where $\partial / \partial t$ acts only on $P(x)(5.4)$. Some properties of these integrals can be found in refs. [16].

For the running of $e^{2}(k)$ or, equivalently. for the anomalous dimension $\eta_{F}$ one finds an answer of the form

$$
\begin{aligned}
\frac{\partial}{\partial t} e^{2} & =\left(d-4+\eta_{F}\right) e^{2} \\
& \equiv(d-4) e^{2}+\frac{4}{3} v_{d} e^{t}\left(l_{g}^{d} \bar{s}_{g}^{d}\left(2 \lambda \kappa, 2 e^{2} \kappa\right)+l_{c}^{d}\right)
\end{aligned}
$$



$(4.14)$

Here the constant $l_{c}^{d}$ arises from the term $\frac{\partial}{\partial t} C_{k}$ in (5.1). For $d>2$ it follows from

$$
l_{c}^{d}=\frac{d-2}{4} k^{4-d} \int_{0}^{\infty} d x x^{\frac{d-4}{2}} \frac{d}{d x} \frac{d}{d t} \ln \left(1+\frac{P-x}{k^{2}}\right)
$$

and for $d=2$ it is given by

$$
l_{c}^{2}=\frac{1}{2} k^{2} \frac{d}{d x} \frac{d}{d t} \ln \left(1+\frac{P-x}{k^{2}}\right)_{\mid x=0}
$$

With $f_{k}^{2}(x)=\exp \left(-x / k^{2}\right)$ one finds $l_{c}^{4}=0$ and

$$
l_{c}^{2}=\frac{1}{4}
$$

The contribution $\sim l_{g}^{d} \tilde{s}_{g}^{d}$ is related to the one-loop diagrams shown in fig. 1 and evaluated in the two appendices. We first deal in appendix A with the simpler case where $\lambda \ll e^{2}$ and the mass of the scalar field in the loop can be neglected. In this limit one obtains for $d>2$

$$
l_{g}^{d} \tilde{s}_{g}^{d}\left(0,2 e^{2} \kappa\right)=l_{g}^{d}+\frac{48}{d} \frac{d+1}{d+2} e^{2} \kappa\left[m_{2,2}^{d}\left(0,2 e^{2} \kappa\right)-\frac{1}{d+1} n_{2,1}^{d-2}\left(0,2 e^{2} \kappa\right)\right]
$$

where the dimensionless integrals $n_{n_{1}, n_{2}}^{d}$ are given by

$$
n_{n_{1}, n_{2}}^{d}\left(w_{1}, w_{2}\right)=-\frac{1}{2} k^{2\left(n_{1}+n_{2}-1\right)-d} \int_{0}^{\infty} d x x^{\frac{d}{2}} \frac{\partial}{\partial t}\left[\frac{d P}{d x}\left(P+w_{1} k^{2}\right)^{-n_{1}}\left(P+w_{2} k^{2}\right)^{-n_{2}}\right]
$$

The threshold function $\tilde{s}_{g}^{d}$ is normalized such that $\tilde{s}_{g}^{d}(0,0)=1$, and we observe that $\tilde{s}_{g}^{d}\left(0,2 e^{2} \kappa\right) \rightarrow 1$ in the limit $2 e^{2} \kappa \rightarrow \infty$. The non-zero value of $\tilde{s}_{g}^{d}$ even for infinite photon mass $2 e^{2} \kappa k^{2}$ is due to the fact that the scalar degrees of freedom remain massless in this limit and contribute to the running of $e^{2}$ (cf. fig. 1a). The constant $l_{g}^{d}$ reads for $d>2$ (cf. $($ A. 6$)$ )

$$
l_{g}^{d}=-\frac{d-2}{4} k^{4-d} \int_{0}^{\infty} d x x^{\frac{d-t}{2}} \frac{d}{d x}\left[\frac{1}{P} \frac{\partial P}{\partial t}\right]
$$

and one finds, in particular

$$
l_{3}^{4}=1
$$

Neglecting the mass terms of particles in the loop this approximation reproduces the standard running of the four-dimensional gauge coupling in the one-loop approximation. Our nonperturbative result goes, however, beyond the one-loop approximation as demonstrated by the additional $e^{2}$ dependence in $\partial e^{2} / \partial t$ arising from the threshold function

$$
\tilde{s}_{g}^{4}=1+10 e^{2} \kappa\left[m_{2,2}^{4}\left(0,2 e^{2} \kappa\right)-\frac{1}{5} n_{2,1}^{3}\left(0,2 e^{2} \kappa\right)\right]
$$

In appendix $\mathrm{B}$ we give details on the threshold function $\tilde{s}_{g}^{d}\left(2 \lambda \kappa, 2 e^{2} \kappa\right)$ for arbitrary values of $\lambda$ and $e^{2}$. We find, in a good approximation

$\tilde{s}_{g}^{d}\left(2 \lambda \kappa, 2 e^{2} \kappa\right)=\frac{m_{2,2}^{d+2}(2 \lambda \kappa, 0)}{m_{4}^{d+2}}+\frac{48 e^{2} \kappa}{d(d+2) l_{g}^{d}}\left[(d+1) m_{2,2}^{d}\left(2 \lambda \kappa, 2 e^{2} \kappa\right)-n_{2,1}^{d-2}\left(2 \lambda \kappa, 2 e^{2} \kappa\right)\right]$ 
We see how for $\lambda \kappa \gg 1$ the threshold function vanishes. In this limit the heavy scalar fields decouple and do not contribute anymore to the running of the gauge coupling.

It is instructive to expand the $\beta$-function for small values of $e^{2}$ for the scaling solution. (For $e^{2} \rightarrow 0$ the theory becomes a scalar theory with small perturbations and therefore has approximately the standard second-order phase transition of scalar theories.) In lowest order in $e^{2}$ we can replace $\kappa$ by its fixpoint value from (5.9), i.e. $\left(d-2+\eta_{\varphi}\right) \kappa_{*}=2 v_{d} l_{1}^{d}\left(1+3 s_{1}^{d}(2 \lambda \kappa)\right) .^{\top}$ In four dimensions and for small $\lambda$ we expand $\tilde{s}_{g}^{4}\left(0,2 e^{2} \kappa\right)$ of (??) in lowest order in $e^{2}$ and obtain from (5.16) in order $e^{6}$

$$
\begin{aligned}
\beta_{e^{2}}=\frac{\partial e^{2}}{\partial t} & =\frac{2}{3} \frac{e^{4}}{16 \pi^{2}}\left[1+20 l_{1}^{4}\left(m_{4}^{4}-\frac{1}{5} n_{3}^{2}\right) \frac{e^{2}}{16 \pi^{2}}-\frac{1}{2} \eta_{\varphi}\right] \\
& =\frac{2}{3} \frac{e^{4}}{16 \pi^{2}}+\left(\frac{26}{3}-\frac{8}{3} \ln 2\right) \frac{e^{6}}{\left(16 \pi^{2}\right)^{2}}
\end{aligned}
$$

where we have used $l_{1}^{4}=1, m_{4}^{4}=m_{2,2}^{4}(0,0)=\frac{1}{2}, n_{3}^{2}=n_{2,1}^{2}(0,0)=\ln 2$ for $f_{k}^{2}=$ $\exp \left(-x / k^{2}\right)$. We have also included in (5.26) the correction (A.9) with $\eta_{\varphi}=-\frac{3}{8 \pi^{2}} e^{2}$. Numerically, the coefficient of the term $\sim e^{6} /\left(16 \pi^{2}\right)^{2}$ is 6.82 . This is about $85 \%$ of the coefficient 8 known from two-loop perturbation theory for a massless complex scalar field [17]. We conclude that for small $e^{2}$ the dominant effects beyond the one-loop aapproximation are already encoded in the threshold functions.

Now we turn to the symmetric regime of the theory. In this case $\rho_{0}$ vanishes, and instead of $\kappa$ we use the dimensionless, renormalized mass of the scalar, $\tilde{m}^{2}(k)$, in order to parametrize the renormalization group flow. In analogy with eq. (5.7) we introduce the quantities

$$
\begin{aligned}
\tilde{m}^{2}(k) & =k^{-2} Z_{\varphi, k}^{-1} U_{k}^{\prime}(0) \\
\lambda(k) & =k^{d-4} Z_{\varphi, k}^{-2} U_{k}^{\prime \prime}(0) \\
e^{2}(k) & =k^{d-4} Z_{F, k}^{-1} \bar{e}^{-2}
\end{aligned}
$$

The running of $\tilde{m}^{2}$ and $\lambda$ is governed by the equations

$$
\begin{aligned}
\frac{d}{d t} \tilde{m}^{2} & =\left(-2+\eta_{\varphi}\right) \tilde{m}^{2}-4 l_{1}^{d} v_{d}\left[(d-1) e^{2}+2 \lambda s_{1}^{d}\left(\tilde{m}^{2}\right)\right] \\
\frac{d}{d t} \lambda & =\left(d-4+\eta_{\varphi}\right) \lambda+4 l_{2}^{d} v_{d}\left[2(d-1) e^{4}+5 \lambda^{2} s_{2}^{d}\left(\tilde{m}^{2}\right)\right]
\end{aligned}
$$

where the anomalous dimension of the scalar is given by

$$
\eta_{\varphi}=-16 v_{d}\left(1-d^{-1}\right) e^{2} l_{1,1}^{d}\left(0, \tilde{m}^{2}\right)
$$

The equation for the evolution of $e^{2}$ is by far simpler than in the spontaneously broken case. If we write, to lowest order,

$$
\begin{aligned}
\frac{d}{d t} e^{2} & =\left(d-4+\eta_{F}\right) e^{2} \\
& \equiv(d-4) e^{2}+\frac{4}{3} v_{d} l_{g}^{d} s_{\jmath}^{d}\left(\tilde{m}^{2}\right) e^{4}
\end{aligned}
$$

\footnotetext{
${ }^{7}$ See refs. [4],[16] for a detailed discussion of the scaling solution and its relation to the massless $\beta$-function.
} 
then the threshold function $s_{g}^{d}$ reads (for $d>2$ )

$$
l_{g}^{d} s_{g}^{d}\left(\tilde{m}^{2}\right)=-\frac{d-2}{4} k^{4-d} \int_{0}^{\infty} d x x^{\frac{d}{2}-2} \frac{d}{d x}\left[\frac{1}{P+\dot{m}^{2} k^{2}} \frac{\partial}{\partial t} P\right]
$$

For $d=4, l_{g}^{4}=1$, one obtains

$$
s_{g}^{4}\left(\tilde{m}^{2}\right)=\frac{1}{1+\check{m}^{2}}, \quad \text { i.e. } \quad \eta_{F}=\frac{e^{2}}{24 \pi^{2}} \frac{1}{1+\check{m}^{2}}
$$

We see that $s_{g}^{d}\left(\tilde{m}^{2}\right)$ vanishes for $\tilde{m}^{2} \gg 1$. In this limit the heavy scalars decouple and do not renormalize the gauge coupling anymore.

\section{Conclusions and Discussion}

We have presented gauge-invariant exact evolution equations for scalar electrodynamics. They describe the scale dependence of the effective average action $\Gamma_{k}$. The effective average action is a particular type of "coarse-grained action" which obtains by integrating out all quantum fluctuations with momenta (or covariant momenta) $q^{2}>k^{2}$. Despite the close analogy with perturbative one-loop formulae our equations are exact nonperturbative expressions. The momentum integrals appearing in the "nonperturbative loop integrals" are infrared and ultraviolet finite. No explicit ultraviolet regularization has to be specified in order to follow the $k$-dependence of $\Gamma_{k}$. In the limit $k \rightarrow 0$ the effective average action becomes the generating functional for the 1PI Green functions in the background field formalism.

Even though our flow equations are exact, their solution will require approximations. In practice this means that the most general possible form of $\Gamma_{k}$, which is consistent with the symmetries, must be truncated by using an ansatz involving only a small number of parameters. The practical use of our equations for nonperturbative problems mainly depends on the ability to find a viable nonperturbative truncation. For this reason we have put great emphasis in the present paper to maintain gauge invariance at every step. Without gauge invariance the number of possible invariants increases dramatically. Furthermore, it becomes difficult to judge if a given truncation remains within the universality class appropriate for scalar electrodynamics. This problem is related to the well-known fact that the effective action with gauge symmetry is not an infrared stable fixpoint in the space of possible actions with spin zero and spin-one bosons. (For example, a mass term for the transversal spin-one boson is typically generated in a theory without gauge invariance.) The constraints on the $k$-dependent gauge-fixing term discussed in sect. 3 are crucial in this respect. They should be employed in order to guarantee the usual Ward identities for $k=0$ on a given level of truncation. (Without truncations the Ward identities are a consequence of gauge invariance being maintained formally at every step. With a given truncation, however, the absence of a mass term for the transversal photon is not immediately obvious for versions of the flow equation without explicit gauge-invariance, cf. sect. 3.) The price for maintaining 
gauge invariance are correction terms from the $k$-dependence of the effective gaugefixing term. The general form of these correction terms can be rather complicated. Fortunately, the dominant effect can be expressed in terms of a relatively simple determinant whereas the remainder can be treated as a small perturbation (cf. sect. 4).

We have tested the nonperturbative content of our equations by computing the flow equations for the scalar potential and the gauge coupling in scalar electrodynamics in arbitrary dimension $d$. For small couplings and $d=4$ the flow equations reduce in lowest order to the perturbative renormalization group equations in the one-loop approximation. In our rough approximation they also give the two-loop coefficient with an accuracy of $15 \%$ - this without ever performing a two-loop calculation and with the simplest possible truncation of $\Gamma_{k}$. The nonperturbative information is contained here mainly in the appearance of threshold functions describing the decoupling of heavy particles. A more impressive test is the result for $d=3$ where strong renormalization effects drive the renormalized gauge coupling $e_{R}$ to zero proportional to $k^{1 / 2}$ if the theory is in the scaling region. This result cannot be obtained perturbatively. It fully confirms similar findings obtained earlier [8] without the use of exact flow equations. In particular, the (approximate) infrared fixpoint for the dimensionless three-dimensional gauge coupling $e^{2}=e_{R}^{2} / k$ in the region of small $e^{2} \kappa, \lambda \kappa$ or constant $\lambda, \kappa$ can be computed from eqs. (5.16), (5.17), (5.23), and (5.25). It is roughly of the order $6 \pi^{2}$, demonstrating that even $e^{2} / 16 \pi^{2}$ is not a small quantity. The expansion of $\beta_{e^{2}}$ in powers of $e^{2}$ converges badly at the fixpoint. We emphasize that the infrared divergences present in the three-dimensional loop expansion for massless fields are completely under control in our approach. Our approximations can be improved in a straightforward manner by including more terms in the truncation of $\Gamma_{k}$.

As an illustration of practical applications of our nonperturbative evolution equation we briefly discuss the Coleman-Weinberg phase transition [1] in four dimensions. We concentrate on the linear regime of small masses, i.e. $2 \lambda \kappa \ll 1,2 e^{2} \kappa \ll 1$. In this approximation we recover in lowest order in $e^{2}$ and $\lambda$ the usual one-loop $\beta$ functions

$$
\begin{gathered}
\frac{\partial e^{2}}{\partial t}=\frac{e^{4}}{2+\pi^{2}} \\
\frac{\partial \lambda}{\partial t}=\frac{3}{4 \pi^{2}}\left(e^{4}-\lambda e^{2}+\frac{5}{6} \lambda^{2}\right)
\end{gathered}
$$

For small $e^{2}$ we can neglect the slow decrease of $e^{2}(k)$ for decreasing $k$ and take $e^{2}$ constant. Then $\lambda$ is driven to zero at some scale $k_{c}>0$. We only consider the last part of the running where $\lambda \ll e^{2}$ and

$$
\begin{gathered}
\lambda(k)=\frac{3}{4 \pi^{2}} e^{4} \ln \frac{k}{k_{\varepsilon}} \\
k_{c}=\Lambda \exp \left(-\frac{4 \pi^{2}}{3 e^{4}} \lambda(.1)\right)
\end{gathered}
$$

Here $\Lambda$ is some short-distance scale where the hierarchy of couplings $\lambda \ll e^{2} \ll 1$ is valid. In addition to eqs. (6.1) and (6.2) our approach also provides an equation for 
the $k$-dependence of the potential minimum

$$
\frac{\partial}{\partial t} \kappa=-2 \kappa+\frac{1}{4 \pi^{2}}-\frac{3}{8 \pi^{2}} \lambda \kappa+\frac{3}{8 \pi^{2}} \frac{e^{2}}{\lambda}-\frac{3}{4 \pi^{2}} \frac{e^{4} \kappa}{\lambda}+\frac{3}{8 \pi^{2}} e^{2} \kappa
$$

We observe the appearance of factors $\lambda^{-1}$ which reflect a fast change of the location of the minimum in regions where $\lambda$ and therefore the curvature around the minimum is small. For $\lambda \ll e^{2} \ll 1$ the solution for $\kappa$ is determined by an infrared fixpoint in the dimensionless scalar mass term $\lambda \kappa$ :

$$
\begin{gathered}
\lambda(k) \kappa(k) \rightarrow \lambda_{\approx} \kappa_{*}=\frac{3}{16 \pi^{2}} e^{2} \\
\kappa(k)=\frac{1}{4 e^{2}} \frac{1+\Delta_{\Lambda} \frac{\Lambda^{2}}{k^{2}}}{\ln k / k_{c}} \\
\Delta_{\Lambda}=\frac{\lambda(\Lambda) \kappa(\Lambda)}{\lambda_{\star} \kappa_{*}}-1
\end{gathered}
$$

The solution (6.7) for $\kappa(k)$ clearly exhibits a bifurcation phenomenon. Depending on the initial value specified by $\Delta_{\Lambda}$ we see that $\kappa(k)$ either becomes zero for $k>k_{c}$ (symmetric phase) or it diverges for $k \rightarrow k_{c}$ (phase with spontaneous symmetry breaking). This bifurcation phenomenon is different from the typical behaviour of $\kappa$ for a second-order phase transition. At first sight it suggests a first-order phase transition as indicated by the perturbative analysis [18]. We emphasize, however, that in the interesting region the validity of the linear approximation is not given since $2 e^{2} \kappa \ll 1$ is violated. A nonperturbative study of the character of the ColemanWeinberg phase transition should use a numerical integration of the evolution equations derived in the last section and is in progress [19].

The nonperturbative character of our flow equations allows one to address the problem of the phase transition of the abelian Higgs model in three dimensions. This should be relevant for normal superconductivity. A successful nonperturbative test of our equations also opens the door for a nonperturbative treatment of the high temperature phase transition in four-dimensional scalar electrodynamics. This should be described by solving the flow equations presented in sect. 5 using appropriately modified temperature-dependent momentum integrals in analogy with the work of ref. [5].

\section{Appendix A}

In this appendix we derive eq. (5.1T) for the threshold function $\tilde{s}_{g}^{d}\left(0,2 e^{2} \kappa\right)$, which is relevant in the regime where the photon mass $2 e^{2} \kappa k^{2}$ is much larger than the (radial) scalar mass $2 \lambda \kappa k^{2}$. This approximation leads to a significant simplification of the quadratic action (5.5) because it eliminates the terms containing $(\delta \varphi)^{2}$ and $\left(\delta \varphi^{*}\right)^{2}$. In order to implement the gauge choice $\alpha=0$ we decompose $\delta A_{\mu}$ according to ${ }^{8}$

$$
\delta A_{\mu}=t_{\mu}+\sqrt{\alpha} \partial_{\mu}\left(-\partial^{2}\right)^{-1 / 2} \tilde{l}, \quad \partial^{\mu} t_{\mu}=0,
$$

\footnotetext{
${ }^{8}$ The longitudinal component $\tilde{l}$ is related to the field $l$ used in $[\bar{l}],[8]$ by $\tilde{l}=\left(\frac{-\partial^{2}}{\alpha}\right)^{1 / 2} l$.
} 
and let $\alpha \rightarrow 0$ in eq. (5.5). This leads to a further simplification because the longitudinal gauge field $\tilde{l}$ decouples:

$$
\begin{aligned}
& \frac{1}{2} \int d^{d} x \delta \psi\left[\Gamma_{k}^{(2)}+\hat{R}_{k}\right] \delta \psi \\
& =\int d^{d} x\left\{\frac{1}{2} t_{\mu}\left[Z_{F, k} P\left(-\partial^{2}\right)+2 Z_{\varphi, k} \bar{e}^{2} \rho_{0}\right] t^{\mu}+\frac{1}{2} \tilde{l} P\left(-\partial^{2}\right) \tilde{l}\right. \\
& +2 \bar{e}^{2} \sqrt{\rho_{0}} Z_{\varphi, k} t_{\mu} A^{\mu}\left(\delta \varphi+\delta \varphi^{*}\right) \\
& \left.+Z_{\varphi, k} \delta \varphi^{*} P\left(-D^{2}\right) \delta \varphi\right\}
\end{aligned}
$$

Here we have set $\varphi=\varphi^{*}=$ const $\equiv \rho_{0}^{1 / 2}$ since the anomalous dimension $\eta_{F}$ which we are going to compute is defined in terms of $Z_{F, k} \equiv Z_{F, k}\left(\rho=\rho_{0}\right)$. We write

$$
\frac{1}{2} \operatorname{Tr}\left[\left(\Gamma_{k}^{(2)}+R_{k}\right) \partial_{t} R_{k}\right]=\frac{\partial}{\partial t}\left(\gamma_{0}+\gamma_{1}[A]+\gamma_{2}[A]\right)
$$

with $\gamma_{0}$ independent of $A$ and obtain by expanding the trace

$$
\begin{gathered}
\gamma_{1}[A]=\operatorname{Tr} \ln \left[P\left(-D^{2}\right)\right] \\
\gamma_{2}[A]=4 \kappa e^{4} k^{6-d} \operatorname{Tr}\left[\frac{P_{T}}{P\left(-\partial^{2}\right)+2 e^{2} \kappa k^{2}} \cdot A^{\mu} \frac{1}{P\left(-\partial^{2}\right)} A_{\mu}\right]+\ldots
\end{gathered}
$$

Here the dots stand for higher powers of $A_{\mu}$, and $P_{T}$ denotes the transverse projector. The contribution (A.4) is most easily evaluated by means of the magnetic field method which we described in ref. [\&]. The result is (for $d>2$ )

$$
\eta_{F 1}=-v_{d-2} \frac{e^{2}}{6 \pi} k^{4-d} \int_{0}^{\infty} d x x^{\frac{d}{2}-2} \frac{d}{d x}\left[\frac{1}{P} \frac{\partial}{\partial t} P\right]
$$

By a standard calculation one can express the part of (A.5) giving rise to the $F_{\mu \nu} F^{\mu \nu}$. term in terms of the integrals (5.15), (5.21):

$$
\eta_{F 2}=\frac{64 v_{d}}{d(d+2)} e^{4} \kappa\left[(d+1) m_{2.2}^{d}\left(0.2 \epsilon^{2} \kappa\right)-n_{2.1}^{d-2}\left(0.2 e^{2} \kappa\right)\right]
$$

Combining (A.6) with (A.T) and using eq. (5.16) for the definition of $\tilde{s}_{g}^{d}$ in terms of $\eta_{F}$ we obtain the threshold function (5.20) given in the main text.

One word of caution is necessary here concerning the use of the symbol $\partial / \partial t$. From the starting expression (A.1) it follows that $\partial / \partial t$ always appears under the momentum integrals (this guarantees finiteness of all integrals) and should be applied only on $R_{k}$. Only in the approximation where the $k$-dependence of $Z_{\bullet, k}$ and $Z_{F, k}$ in the definition of $R_{k}$ is neglected $\partial / \partial t$ reduces to a derivative acting on $P$. In order to demonstrate how to go beyond this approximation we give the additional 
contribution to $\eta_{F 1}$ from the $k$-dependence of $Z_{\varphi, k}$ appearing in $R_{k}$. This correction is proportional to $\eta_{\varphi}(5.13)$

$$
\Delta \eta_{F 1}=\frac{v_{d-2}}{6 \pi} \eta_{\varphi} e^{2} k^{4-d} \int_{0}^{\infty} d x x^{\frac{d}{2}-2} \frac{d}{d x}\left(\frac{P-x}{P}\right)
$$

and reads for $d=4$

$$
\Delta \eta_{F 1}=-\frac{\eta_{\varphi} e^{2}}{48 \pi^{2}}
$$

\section{Appendix B}

In this appendix we study in some detail the threshold function $\tilde{s}_{g}^{d}\left(2 \lambda \kappa, 2 e^{2} \kappa\right)$ for arbitrary values of $\lambda$ and $e^{2}$. From eqs. (5.3), (5.8) and (5.16) it is obvious that $\tilde{s}_{g}^{d}$ can be extracted from the derivative part of the second functional derivative of $\Gamma_{k}$ with respect to $A_{\mu}$ :

$$
\begin{aligned}
\left.\frac{\delta^{2} \Gamma_{k}[\varphi, A]}{\delta A_{\alpha}(y) \delta A_{\beta}(z)}\right|_{A=0} \equiv \int \frac{d^{d} Q}{(2 \pi)^{d}} Z_{F, k}\left(\rho, Q^{2}\right) \cdot \\
\cdot\left(Q^{2} \delta^{\alpha \beta}-Q^{\alpha} Q^{\beta}\right) e^{-i Q(y-z)}+\ldots
\end{aligned}
$$

Here $\varphi \equiv \rho^{1 / 2}$ is chosen to be an $x^{\mu}$-independent constant. The wave function renormalization $Z_{F, k}$ which determines $\tilde{s}_{g}^{d}$ obtains as

$$
Z_{F, k}=Z_{F, k}\left(\rho_{0}, Q^{2}=0\right)
$$

where $\rho_{0}$ denotes the minimum of the potential $U_{k}(\rho)$. Taking two functional derivatives of eq. (5.6) we obtain

$$
\begin{aligned}
& \left.\frac{\partial}{\partial t} \frac{\delta^{2} \Gamma_{k}[\varphi, A]}{\delta A_{\alpha}(y) \delta A_{\beta}(z)}\right|_{A=0} \\
& =-\frac{1}{2} \frac{\partial}{\partial t} \operatorname{Tr}\left[G \frac{\delta \hat{\Gamma}_{k}^{(2)}}{\delta A_{\beta}(z)} G \frac{\delta \hat{\Gamma}_{k}^{(2)}}{\delta A_{\alpha}(y)}\right]_{A=0} \\
& +\frac{1}{2} \frac{\partial}{\partial t} \operatorname{Tr}\left[G \frac{\delta^{2} \hat{\Gamma}_{k}^{(2)}}{\delta A_{\alpha}(y) \delta A_{\beta}(z)}\right]_{A=0}
\end{aligned}
$$

In this equation the complete kinetic operator

$$
\hat{\Gamma}_{k}^{(2)} \equiv \Gamma_{k}^{(2)}+\hat{R}_{k} \equiv \Gamma_{k}^{(2)}+R_{k}+S_{g f}^{(2)}
$$

includes the infrared cutoff term $R_{k}$ and the gauge-fixing $S_{g f}^{(2)}$ and $\left.G=\left(\hat{\Gamma}_{k}^{(2)}\right)\right)^{-1}$ denotes its inverse. (In writing down eq. (B.3) we omitted the term stemming from $C_{k}[A]$. It is independent of $\kappa$ and has already been given in eq. (5.17).) The inverse effective propagator $\hat{\Gamma}_{k}^{2}$ can be read off from the quadratic form (5.5) with 
$\delta A_{\mu}$ decomposed according to eq. (A.1). It will prove useful to choose $\varphi$ real and to express the scalar fluctuation as

$$
\delta \varphi=2^{-1 / 2}(\sigma+i \omega)
$$

in terms of two real fields $\sigma$ and $\omega$. They correspond to the radial ("Higgs") and angular ("Goldstone") excitations, respectively. Thus (5.5) becomes

$$
\begin{aligned}
& \int d^{d} x \delta \psi \hat{\Gamma}_{k}^{(2)} \delta \psi \\
& =\int d^{d} x\left\{t_{\mu}\left[Z_{F, k} P_{F}\left(-\partial^{2}\right)+2 \vec{e}^{2} Z_{\varphi, k} \rho\right] t^{\mu}+\tilde{l} P_{F}\left(-\partial^{2}\right) \tilde{l}\right. \\
& +\sigma\left[Z_{\varphi, k} P_{\varphi}\left(-D^{2}\right)_{s y m}+U_{k}^{\prime}(\rho)+2 \rho U_{k}^{\prime \prime}(\rho)\right] \sigma \\
& +\omega\left[Z_{\varphi, k} P_{\varphi}\left(-D^{2}\right)_{s y m}+U_{k}^{\prime}(\rho)\right] \omega \\
& +2 Z_{\varphi, k} \omega P_{\varphi}\left(-D^{2}\right)_{a s} \sigma \\
& \left.+4 \sqrt{2} \bar{e}^{2} \sqrt{\rho} Z_{\varphi, k} t_{\mu} A^{\mu} \sigma\right\}
\end{aligned}
$$

with the (anti-)symmetric combinations

$$
\begin{aligned}
P_{\varphi}\left(-D^{2}\right)_{s y m} & =\frac{1}{2}\left[P_{\varphi}\left(-D^{2}(A)\right)+P_{\varphi}\left(-D^{2}(-A)\right)\right] \\
P_{\varphi}\left(-D^{2}\right)_{a s} & =\frac{1}{2 i}\left[P_{\varphi}\left(-D^{2}(A)\right)-P_{\varphi}\left(-D^{2}(-A)\right)\right]
\end{aligned}
$$

The notations $P_{F}$ and $P_{\varphi}$ indicate that we may use different cutoff functions $R_{k}$ for the photon and for the scalar, respectively. In order to evaluate the traces on the r.h.s. of (B.3), we need the components of the propagator $G$ and the generalized vertices $\delta \hat{\Gamma}_{k}^{(2)} / \delta A_{\alpha}$ and $\delta^{2} \hat{\Gamma}_{k}^{(2)} / \delta A_{\alpha} \delta A_{\beta}$ for $A_{\mu}=0$ only. For $A_{\mu}=0$ the quadratic form (B.6) is diagonal, and we can easily read off the propagators

$$
\begin{aligned}
& G_{t t}^{\mu \nu}\left(x_{1}, x_{2}\right)=\left[Z_{F, k} P_{F}\left(-\partial_{1}^{2}\right)+2 \bar{e}^{2} Z_{\varphi, k} \rho\right]^{-1}\left(\delta^{\mu \nu}-\partial_{1}^{\mu} \partial_{1}^{\nu} / \partial_{1}^{2}\right) \delta\left(x_{1}-x_{2}\right) \\
& G_{\sigma \sigma}\left(x_{1}, x_{2}\right)=\left[Z_{\varphi, k} P_{\varphi}\left(-\partial_{1}^{2}\right)+U_{k}^{\prime}+2 \rho U_{k}^{\prime \prime}\right]^{-1} \delta\left(x_{1}-x_{2}\right) \\
& G_{\omega \omega}\left(x_{1}, x_{2}\right)=\left[Z_{\varphi, k} P_{\varphi}\left(-\partial_{1}^{2}\right)+U_{k}^{\prime}\right]^{-1} \delta\left(x_{1}-x_{2}\right)
\end{aligned}
$$

(The field $\tilde{l}$ decouples for the gauge choice $\alpha \rightarrow 0$ and is discarded in the following.) The couplings among $t_{\mu}, \sigma$ and $\omega$ involve the following matrix elements of $\hat{\Gamma}_{k}^{(2)}$ :

$$
\begin{aligned}
\Gamma_{t \sigma}^{\mu}\left(x_{1}, x_{2}\right) & =\Gamma_{\sigma t}^{\mu}\left(x_{1}, x_{2}\right)=2 \sqrt{2} \bar{e}^{2} \sqrt{\rho} Z_{\nu, k} A^{\mu}\left(x_{1}\right) \delta\left(x_{1}-x_{2}\right) \\
\Gamma_{\omega \sigma}\left(x_{1}, x_{2}\right) & =-\Gamma_{\sigma \omega}\left(x_{1}, x_{2}\right)=Z_{\phi, k} P_{\vartheta}\left(-D_{1}^{2}\right)_{\alpha s} \delta\left(x_{1}-x_{2}\right)
\end{aligned}
$$

They give rise to the vertices

$$
\begin{aligned}
& \left.\frac{\delta \Gamma_{t \sigma}^{\mu}\left(x_{1}, x_{2}\right)}{\delta A_{\alpha}(y)}\right|_{A=0}=\left.\frac{\delta \Gamma_{\sigma t}^{\mu}\left(x_{1}, x_{2}\right)}{\delta A_{\alpha}(y)}\right|_{A=0}=2 \sqrt{2} \bar{e}^{2} \sqrt{\rho} Z_{\omega, k} \delta^{\alpha \mu} \delta\left(y-x_{1}\right) \delta\left(y-x_{2}\right) \\
& \left.\frac{\delta \Gamma_{\omega \sigma}\left(x_{1}, x_{2}\right)}{\delta A_{\alpha}(y)}\right|_{A=0}=-\left.\frac{\delta \Gamma_{\sigma \omega}\left(x_{1}, x_{2}\right)}{\delta A_{\alpha}(y)}\right|_{A=0}=-2 \bar{e} Z_{\nu, k}\left[\dot{P}_{\varphi}\left(-\partial_{y}^{2}\right) \delta\left(y-x_{1}\right)\right]\left[\partial_{y}^{\alpha} \delta\left(y-x_{2}\right)\right]
\end{aligned}
$$




$$
\begin{aligned}
& \left.\frac{\delta \Gamma_{\sigma \sigma}\left(x_{1}, x_{2}\right)}{\delta A_{\alpha}(y) \delta A_{\beta}(z)}\right|_{A=0}=\left.\frac{\delta \Gamma_{\psi \omega}\left(x_{1}, x_{2}\right)}{\delta A_{\alpha}(y) \delta A_{\beta}(z)}\right|_{A=0} \\
& =2 Z_{\varphi, k} \bar{e}^{2} \delta^{\alpha \beta} \dot{P}_{\varphi}\left(-\partial_{1}^{2}\right) \delta\left(x_{1}-y\right) \delta\left(x_{1}-z\right) \delta\left(x_{1}-x_{2}\right)+O\left(\ddot{P}_{\varphi}\right)
\end{aligned}
$$

where we write $\Gamma_{\omega \sigma} \equiv\left(\hat{\Gamma}_{k}^{(2)}\right)_{\omega \sigma}, \ldots$ for the various components. The terms involving second derivatives $\ddot{P}_{\varphi}(x) \equiv d^{2} R_{k, \varphi}(x) / d x^{2}$ are rather complicated and we shall not consider them here. This approximation is justified if $x \bar{P}_{\varphi} \ll \dot{P}_{\varphi}$. It becomes exact for cutoff functions which are independent of $x$, i.e., for $R_{k, \varphi}=Z_{\varphi, k} k^{2}$ and $\dot{P}_{\varphi}=1$. Using the above propagators and vertices, (B.3) reads

$$
\left.\frac{\partial}{\partial t} \frac{\delta^{2} \Gamma_{k}[\varphi, A]}{\delta A_{\alpha}(y) \delta A_{\beta}(z)}\right|_{A=0}=T_{(a)}^{\alpha \beta}(y, z)+T_{(b)}^{\alpha \beta}(y, z)+T_{(c)}^{\alpha \beta}(y, z)
$$

with the terms

$$
\begin{aligned}
& T_{(a)}^{\alpha \beta}(y, z)=-\frac{1}{2} \frac{\partial}{\partial t} \operatorname{Tr}\left[G_{\sigma \sigma} \frac{\delta \Gamma_{\sigma \omega}}{\delta A_{\alpha}(y)} G_{\omega \omega} \frac{\delta \Gamma_{\omega \sigma}}{\delta A_{\beta}(z)}\right] \\
&+(\alpha \leftrightarrow \beta, y \leftrightarrow z) \\
& T_{(b)}^{\alpha \beta}(y, z)=-\frac{1}{2} \frac{\partial}{\partial t} \operatorname{Tr}\left[G_{t t} \frac{\delta \Gamma_{t \sigma}}{\delta A_{\alpha}(y)} G_{\sigma \sigma} \frac{\delta \Gamma_{\sigma t}}{\delta A_{\beta}(z)}\right] \\
&+(\alpha \leftrightarrow \beta, y \leftrightarrow z) \\
& T_{(c)}^{\alpha \beta}(y, z)=\frac{1}{2} \frac{\partial}{\partial t} \operatorname{Tr}\left[G_{\sigma \sigma} \frac{\delta^{2} \Gamma_{\sigma \sigma}}{\delta A_{\alpha}(y) \delta A_{\beta}(z)}\right]+(\sigma \rightarrow \omega)
\end{aligned}
$$

corresponding to the diagrams of Figs. $1 \mathrm{a}, 1 \mathrm{~b}$ and $1 \mathrm{c}$, respectively. (From now on "Tr" denotes the trace over space time coordinates only.) The polarization function $Z_{F, k}\left(\rho, Q^{2}\right)$ of (B.1) writes similarly

$$
Z_{F, k}=Z_{F, k}^{(a)}+Z_{F, k}^{(\bar{a})}+Z_{F, k}^{(b)}+Z_{F, k}^{(c)}
$$

For later convenience we decomposed the first term in two pieces. Using the explicit forms of the propagators and vertices, one finds, with $P_{T}^{\alpha \beta}(Q)=\delta^{\alpha \beta}-Q^{\alpha} Q^{\beta} / Q^{2}$,

$$
\begin{aligned}
& Z_{F, k}^{(a)}\left(\rho, Q^{2}\right) Q^{2} P_{T}^{\gamma \beta}(Q)=-4 \bar{e}^{2} Z_{;, k}^{2} P_{T}^{\gamma} \alpha(Q) \\
& \int \frac{d^{d} q}{(2 \pi)^{d}} \frac{q^{\alpha} q^{\beta}}{\left[Z_{\varphi, k} P_{\varphi}\left(q^{2}\right)+U_{k}^{\prime}(\rho)+2 \rho L_{k}^{\prime \prime \prime}(\rho)\right]\left[Z_{\rho, k} P_{\varphi}\left((q+Q)^{2}\right)+U_{k}^{\prime}(\rho)\right]} \\
& Z_{F, k}^{(\bar{a})}\left(\rho, Q^{2}\right) Q^{2} P_{T}^{\sim \beta}(Q)=-4 \bar{e}^{2} Z_{\varphi, k}^{2} P_{T}^{\sim \prime}(Q) \\
& \cdot \int \frac{d^{d} q}{(2 \pi)^{d}} \frac{\left(\dot{P}_{\rho}\left(q^{2}\right) \dot{P}_{\rho}\left((q+Q)^{2}\right)-1\right) q^{\alpha} q^{\mathcal{\beta}}}{\left[Z_{\rho, k} P_{\varphi}\left(q^{2}\right)+U_{k}^{\prime}(\rho)+2 \rho U_{k}^{\prime \prime}(\rho)\right]\left[Z_{\varphi, k} P_{\rho}\left((q+Q)^{2}\right)+U_{k}^{\prime}(\rho)\right]} \\
& Z_{F, k}^{(b)}\left(\rho, Q^{2}\right) Q^{2} P_{T}^{+\beta}(Q)=-8 \bar{e}^{4} \rho Z_{\hat{\psi}, k}^{2} P_{T \alpha}^{\alpha}(Q) \\
& \cdot \int \frac{d^{d} q}{(2 \pi)^{d}} \frac{\delta^{\alpha \beta}-q^{\alpha} q^{\beta} / q^{2}}{\left[Z_{F, k} P_{F}\left(q^{2}\right)+2 \bar{e}^{2} Z_{\varphi, k} \rho\right]\left[Z_{\rho, k} P_{f}\left((q+Q)^{2}\right)+U_{k}^{\prime}(\rho)+2 \rho U_{k}^{\prime \prime}(\rho)\right]}
\end{aligned}
$$




$$
Z_{F, Q}^{(c)}\left(\rho, Q^{2}\right) Q^{2} P_{T}^{\gamma \beta}(Q)=0+O\left(\tilde{P}_{\bullet}\right)
$$

Apart from neglecting the $O\left(\vec{P}_{\varphi}\right)$ term in (B.21), no approximations have been made yet. Expanding the integrals in (B.18) and (B.20) to order $Q^{2}$, one finds, for equal but otherwise arbitrary functions $P_{F}(x)=P_{\varphi}(x) \equiv P(x)$

$$
\begin{aligned}
\frac{\partial}{\partial t} Z_{F, k}^{(a)}= & -\frac{32 v_{d}}{d(d+2)} Z_{F, k} e^{2} m_{2,2}^{d+2}(2 \lambda \kappa, 0) \\
\frac{\partial}{\partial t} Z_{F, k}^{(b)}= & -\frac{64 v_{d}}{d} \frac{d+1}{d+2} Z_{F, k} e^{4} \kappa m_{2,2}^{d}\left(2 \lambda \kappa, 2 e^{2} \kappa\right) \\
& +\frac{64 v_{d}}{d(d+2)} Z_{F, k} e^{4} \kappa n_{2,1}^{d-2}\left(2 \lambda \kappa, 2 e^{2} \kappa\right)
\end{aligned}
$$

Here we expressed the results in terms of the dimensionless renormalized parameters (5.7) and the integrals $(5.15),(5.21)$. Furthermore, as we neglect the $\ddot{P}_{\varphi}$-terms in (B.21), we may apply the same approximation to the integral in (B.19) which entails $Z_{F, k}^{(\bar{a})}=0$. Within this approximation, the anomalous dimension reads

$$
\begin{aligned}
\eta_{F}= & -\frac{1}{Z_{F, k}} \frac{\partial}{\partial t}\left(Z_{F, k}^{(a)}+Z_{F, k}^{(b)}\right) \\
& =\frac{32 v_{d}}{d(d+2)} e^{2} m_{2,2}^{d+2}(2 \lambda \kappa, 0) \\
& +\frac{64 v_{d}}{d} \frac{d+1}{d+2} e^{4} \kappa m_{2,2}^{d}\left(2 \lambda \kappa, 2 e^{2} \kappa\right) \\
& -\frac{64 v_{d}}{d(d+2)} e^{4} \kappa n_{2,1}^{d-2}\left(2 \lambda \kappa, 2 e^{2} \kappa\right)
\end{aligned}
$$

The corresponding contribution to the threshold function obtains as $\tilde{s}_{g}^{d}=\left(\frac{4}{3} l_{g}^{d} v_{d} e^{2}\right)^{-1} \eta_{F}$ with $l_{g}^{d}$ given in (5.22). We observe that for $\lambda=0$ we reproduce the results (A.6), (A.7) if $\ddot{P}=0$ (e.g. $P=x+k^{2}$ ). The deviation from (A.6) for general $P(x)$ results from the neglected contributions $Z_{F, k}^{(\hat{a})}+Z_{F, k}^{(c)}$. It vanishes for $d=4$. The threshold function is adapted to reproduce $(A . \bar{T})$ for $\lambda=0$ and we obtain, consistent with our approximation,

$$
\tilde{s}_{g}^{d}\left(2 \lambda \kappa, 2 e^{2} \kappa\right)=\frac{m_{2,2}^{d+2}(2 \lambda \kappa, 0)}{m_{4}^{d+2}}+\frac{48 e^{2} \kappa}{d(d+2) l_{g}^{d}}\left[(d+1) m_{2,2}^{d}\left(2 \lambda \kappa, 2 e^{2} \kappa\right)-n_{2,1}^{d-2}\left(2 \lambda \kappa, 2 e^{2} \kappa\right)\right]
$$

where

$$
m_{n_{1}+n_{2}}^{d}=m_{n_{1}, n_{2}}^{d}(0,0)
$$

with

$$
m_{4}^{6}=1
$$




\section{References}

[1] S. Coleman and E. Weinberg, Phys. Rev. D7 (1973) 1888

[2] D. A. Kirzhnits and A. D. Linde, Phys. Lett. B42 (1972) 471; JETP 40 (1974) 628; Ann. Phys. 101 (1976) 195;

J. Kapusta, Finite Temperature Field Theory, Cambridge University Press (1989)

[3] J. R. Espinosa, M. Quiros, and F. Zwirner, preprint CERN TH-6577-92;

W. Buchmüller, Z. Fodor, and T. Helbig, and D. Walliser, preprint DESY 9321 ;

D. Bödeker, W. Buchmüller, Z. Fodor, and T. Helbig, preprint DESY 93-147

[4] C. Wetterich, Nucl. Phys. B352 (1991) 529;

C. Wetterich, Z. Phys. C57 (1993) 451

[5] N. Tetradis and C. Wetterich, Nucl. Phys. B398 (1993) 659;

N. Tetradis and C. Wetterich, preprint DESY 93-128 and Heidelberg preprint HD-THEP-93-36

[6] J. Langer, Ann. Phys. 41 (1967) 10s; ibid. 54 (1969) 258; Physica 73 (1974) 61

[7] M. Reuter and C. Wetterich, Nucl. Phys. B391 (1993) 147

[8] M. Reuter and C. Wetterich, Nucl. Phys. B408 (1993) 91

[9] C. Wetterich, Phys. Lett. B301 (1993) 90

[10] F. Wegner and A. Houghton, Phys. Rev. As (1973) 401;

K. G. Wilson and I. G. Kogut, Phys. Rep. 12 (1974) 75;

F. Wegner, in Phase Transitions and Critical Phenomena, vol. 6, eds. C. Domb and M. S. Green (Academic Press, New York 1976);

J. F. Nicoll, T. S. Chang, and H. E. Stanley, Phys. Rev. Lett. 33 (1974) 540; Phys. Lett. 57 A (1976) 7;

S. Weinberg, Critical Phenomena for Field Theorist.s. Erice Subnucl. Phys. (1976) 1;

J. F. Nicoll and T. S. Chang, Phys. Lett. 62A (197T) 28T:

K. Kawasaki, T. Imaeda, and J. Gunton, in Perspectives in Statistical Physics, ed. H. Raveché, North Holland (1981):

J. Polchinski, Nucl. Phys. B231 (1984) 269;

A. Hasenfratz and P. Hasenfratz, Nucl. Phys. B270 (1986) 68.5;

P. Hasenfratz and J. Nager, Z. Phys. C37 (1988)47T;

B. Warr, Annals of Physics 183 (1988) 1 and 59;

T. Hurd, Commun. Math. Phys. 124 (1989) 1.53:

G. Keller, C. Kopper, and M. Salmhofer, Helv. Phys. Acta 65 (1992) 32;

G. Keller and C. Kopper, Phys. Lett. B273 (1991) 323: 
U. Ellwanger, Z. Phys. C58 (1993) 619;

U. Ellwanger and L. Vergara, Nucl. Phys. B398 (1993) 52

[11] U. Ellwanger, private communication;

M. Bonini, M. D'Attanasio, and G. Marchesini, Parma preprint URPF 92-360;

C. Wetterich, Heidelberg preprint HD-THEP-93-17, to appear in Int. J. Mod.

Phys. A

[12] G. 't Hooft, Acta Univ. Wratislav, N368 (1976);

B. De Witt, in: Quantum Gravity 2, ed. C. Isham (Oxford UP, 1982);

B. Boulware, Phys. Rev. D23 (1981) 389;

L. F. Abbott, Nucl. Phys. B185 (1981) 189

[13] C. Wetterich, Heidelberg preprint HD-THEP-92-64, to appear in Z. Phys. C

[14] M. Reuter and C. Wetterich, Heidelberg preprint THEP-93-40 and preprint DESY $93-152$

[15] M. Bonini, M. D'Attanasio, and G. Marchesini, Parma preprint UPRF 93-382

[16] N. Tetradis and C. Wetterich, preprint DESY 93-094 and Heidelberg preprint HD-THEP-93-28

[17] D. R. T. Jones, Nucl. Phys. B87 (1975) 127; Phys. Rev. D25 (1982) 58

[18] A. D. Linde, JETP Letters 23 (1976) 64;

S. Weinberg, Phys. Rev. Lett. 36 (1976) 294

[19] D. Litim, N. Tetradis, and C. Wetterich, in preparation.

\section{Figure Caption}

Fig. 1: Diagrams contributing to the running of the gauge coupling 

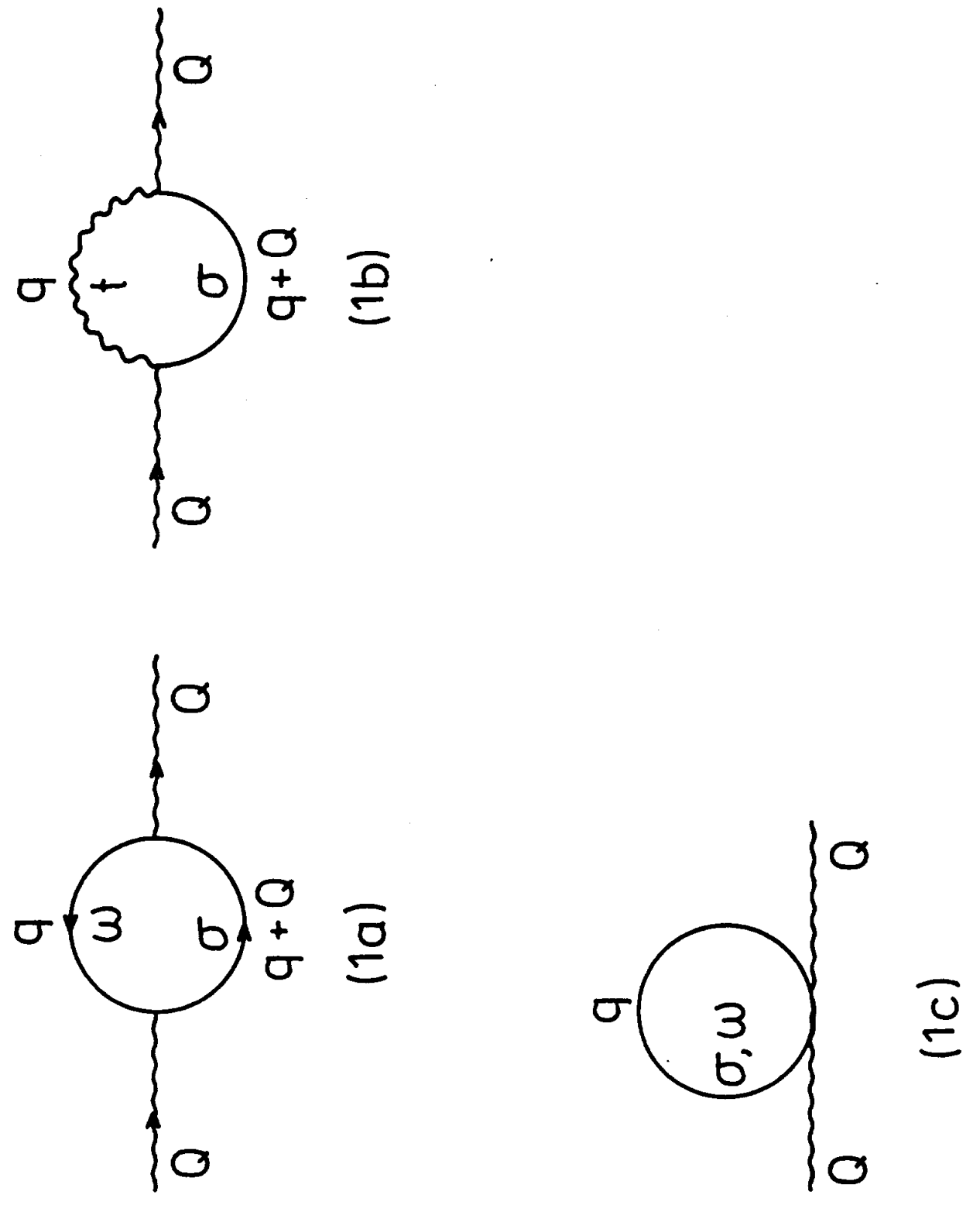
